ARTICLE

https://doi.org/10.1038/s41467-021-20904-2

\title{
NUPR1 is a critical repressor of ferroptosis
}

\author{
Jiao Liu ${ }^{1,12}$, Xinxin Song ${ }^{2,12}$, Feimei Kuang ${ }^{1}$, Qiuhong Zhang ${ }^{3}$, Yangchun Xie ${ }^{4}$, Rui Kang ${ }^{2}$, \\ Guido Kroemer (1) $5,6,7,8,9,10,11 \otimes$ \& Daolin Tang (1) ${ }^{1,2 凶}$
}

Ferroptosis is a type of iron-dependent regulated cell death, representing an emerging disease-modulatory mechanism. Transcription factors play multiple roles in ferroptosis, although the key regulator for ferroptosis in iron metabolism remains elusive. Using NanoString technology, we identify NUPR1, a stress-inducible transcription factor, as a driver of ferroptosis resistance. Mechanistically, NUPR1-mediated LCN2 expression blocks ferroptotic cell death through diminishing iron accumulation and subsequent oxidative damage. Consequently, LCN2 depletion mimics NUPR1 deficiency with respect to ferroptosis induction, whereas transfection-enforced re-expression of LCN2 restores resistance to ferroptosis in NUPR1-deficient cells. Pharmacological or genetic blockade of the NUPR1-LCN2 pathway (using NUPR1 shRNA, LCN2 shRNA, pancreas-specific Lcn2 conditional knockout mice, or the small molecule ZZW-115) increases the activity of the ferroptosis inducer erastin and worsens pancreatitis, in suitable mouse models. These findings suggest a link between NUPR1regulated iron metabolism and ferroptosis susceptibility.

\footnotetext{
${ }^{1}$ The Third Affiliated Hospital, Key Laboratory of Protein Modification and Degradation, Guangzhou Medical University, 510600 Guangdong, China. 2 Department of Surgery, UT Southwestern Medical Center, Dallas, TX 75390, USA. ${ }^{3}$ Department of Surgery, University of Pittsburgh, Pittsburgh, PA 15219, USA. ${ }^{4}$ Department of Oncology, The Second Xiangya Hospital, Central South University, Changsha, Hunan, China. ${ }^{5}$ Université Paris Descartes, Sorbonne Paris Cité, 75006 Paris, France. ${ }^{6}$ Equipe 11 labellisée Ligue Nationale contre le Cancer, Centre de Recherche des Cordeliers, 75006 Paris, France. ${ }^{7}$ Institut National de la Santé et de la Recherche Médicale, U1138 Paris, France. ${ }^{8}$ Université Pierre et Marie Curie, 75006 Paris, France. ${ }^{9}$ Metabolomics and Cell Biology Platforms, Gustave Roussy Cancer Campus, 94800 Villejuif, France. ${ }^{10}$ Pôle de Biologie, Hôpital Européen Georges Pompidou, AP-HP, 75015 Paris, France. ${ }^{11}$ Department of Women's and Children's Health, Karolinska University Hospital, 17176 Stockholm, Sweden. ${ }^{12}$ These authors contributed equally: Jiao Liu, Xinxin Song. ${ }^{凶}$ email: kroemer@orange.fr; daolin.tang@utsouthwestern.edu
} 
C ell death is a fundamental physiological process to maintain homeostasis through the removal of supernumerary, unnecessary, or dysfunctional cells, while pathological death can lead to disease. Unlike accidental cell death, regulated cell death follows multiple subroutines, each of which exhibits distinct molecular cascades and regulatory pathways ${ }^{1,2}$. In recent years, an increasing level of interest has been manifested with regard to ferroptosis, a non-apoptotic-regulated cell death ${ }^{3,4}$, which plays a possible pathogenic role in cancer, neurodegeneration, and organ dysfunction ${ }^{5-7}$. The induction of ferroptosis has been shown to rely on iron accumulation, which facilitates oxidative damage through either the production of highly reactive hydroxyl free radicals in the Fenton reaction or the activation of iron-containing enzymes, such as lipoxygenase ${ }^{8}$. Ferroptotic cells exhibit a necrosis-like morphology and damage-associated molecular patterns (DAMPs) released from ferroptotic cells may function as extracellular inflammatory mediators to contribute to tissue injury ${ }^{9}$.

Ferroptosis is regulated at multiple levels, including at the level of transcription factors that may modulate the resistance of malignant cells to anticancer drugs ${ }^{10}$. Such transcription factors do not only participate in rapid responses to ferroptotic stimuli, but also modulate the long-term outcome of ferroptosis in a context-dependent manner ${ }^{11}$. For example, nuclear factor, erythroid 2-like 2 (NFE2L2/NRF2) serves as a master antioxidant transcription factor for blocking ferroptosis ${ }^{12-14}$, whereas the tumor suppressor TP53 plays a dual role in ferroptosis, depending on the tumor type ${ }^{15-17}$. Although many advances have recently been achieved in the comprehension of antioxidant responses and membrane repair mechanisms ${ }^{18}$, the key transcription factor responsible for controlling iron-dependent ferroptosis has been elusive.

Here, we report that the stress response gene, nuclear protein 1, transcriptional regulator (NUPR1) transactivates the gene encoding lipocalin 2 (LCN2) to diminish iron-induced oxidative damage and to induce ferroptosis resistance. Pharmacological or genetic blockade of the NUPR1-LCN2 pathway may enhance the anticancer activity of ferroptosis activator and pathologic inflammation in vitro and in vivo.

\section{Results}

NUPR1 acts as a repressor of ferroptosis. A number of smallmolecule compounds, including erastin and RSL3, are regularly used to induce ferroptosis and are considered as 'classical' inducers of this regulated cell death subroutine ${ }^{10}$. To identify regulators of ferroptosis, we first evaluated the impact of erastin on the expression of 770 tumor-associated genes in two human pancreatic ductal adenocarcinoma (PDAC) cell lines (PANC1 and BxPC3) using NanoString, a digital technology based on direct multiplexed measurement of nucleic acids through fluorescent barcodes ${ }^{19}$. NUPR1 was identified as one of the top-five erastin-induced genes in both PANC1 and BxPC3 cells (Fig. 1a, b). Quantitative polymerase chain reaction (qPCR) confirmed that both erastin and RSL3 induced the upregulation of NUPR1 mRNA in four human PDAC cell lines (PANC1, BxPC3, Mia$\mathrm{PaCa}$, and CFPAC1), primary human PDAC cells (which we will refer to as "pHsPDAC"), as well as mouse PDAC cell lines (mPDAC) from Pdx1-Cre;K-Ras ${ }^{G 12 D /+}$ mice (Supplementary Fig. 1a). Western blot further confirmed the upregulation of NUPR1 protein expression in PANC1, pHsPDAC, and mPDAC cells in response to erastin or RSL3 (Supplementary Fig. 1b). Endoplasmic reticulum (ER) stress is strongly induced in the context of ferroptosis ${ }^{20}$. Notably, the knockdown of activating transcription factor 4 (ATF4), a key transcription factor involved in ER stress with anti-ferroptosis activity ${ }^{21,22}$, blocked erastin- induced or RSL3-induced NUPR1 mRNA expression in PANC1 cells (Supplementary Fig. 1c, d). These findings indicate that ATF4 facilitates the upregulation of NUPR1 in ferroptosis.

To determine whether NUPR1 is a regulator of ferroptosis, we measured cell viability in wild-type (WT) and Nupr1 $1^{-/-}$mPDAC cells. Nupr1 deletion increased erastin-induced or RSL3-induced growth inhibition (Fig. 1c) and lipid reactive oxygen species (ROS) formation (Fig. 1d) in mPDAC cells, and this effect could be completely reverted by ferroptosis inhibitors (e.g., ferrostatin-1 or liproxstatin-1), but not by inhibitors of apoptosis (e.g., ZVAD-FMK) or necroptosis (e.g., necrosulfonamide). We confirmed these observations in human NUPR1-knockdown PDAC cell lines, including PANC1 and MiaPaCa2 cells (Supplementary Fig. 2). The increased ferroptosis sensitivity was reversed by re-expression of Nupr 1 cDNA in Nupr $1^{-/-}$mPDAC cells (Fig. 1d, e). Collectively, these findings demonstrate that NUPR1 is a negative regulator of ferroptosis.

NUPR1 inhibits iron-dependent oxidative damage in ferroptosis. Iron exists in two oxidation states (ferrous $\left[\mathrm{Fe}^{2}\right]$ or ferric $\left.\left[\mathrm{Fe}^{3+}\right]\right)$, while $\mathrm{Fe}^{2+}$ accumulation is an early signal to initiate ferroptosis ${ }^{8}$. Our biochemical analyses revealed that Nupr1 $1^{-/-}$ mPDAC cells contained higher intracellular $\mathrm{Fe}^{2+}$ levels compared to Nupr $1^{+/+}$cells in response to erastin or RSL3 (Fig. 2a). The increased oxidative stress caused by iron overload may induce ferroptosis through targeting membrane lipids or $\mathrm{DNA}^{23,24}$. Consequently, the depletion of Nupr1 increased erastin-induced or RSL3-induced lipid peroxidation and oxidative DNA damage in mPDAC cells as measured by quantifying malondialdehyde (MDA) or 8-hydroxy-2-deoxy guanosine (8-OHdG), respectively (Fig. 2b, c). As expected, the release of high-mobility group box 1 (HMGB1), a typical DAMP involved in oxidative stress and cell death response ${ }^{9}$, was increased in $N u p r 1^{-1-}$ mPDAC cells following treatment with erastin or RSL3 (Fig. 2d).

Conversely, the iron chelator deferoxamine (DFO) or the antioxidant $\mathrm{N}$-acetylcysteine (NAC) blocked erastin-induced or RSL3-induced cell death in Nupr1-l- mPDAC cells (Fig. 2e), an effect that was associated with decreased production or release of MDA, 8-OHdG, or HMGB1 (Fig. 2b-d). Similarly to Nupr1-/mPDAC cells, NUPR1-knockdown PANC1 cells exhibited increased MDA, 8-OHdG, and HMGB1 release during ferroptotic cell death, which could be reversed by the addition of DFO or NAC (Supplementary Fig. 3). Of note, the shRNA-mediated knockdown of six-transmembrane epithelial antigen of the prostate 3 (Steap3), an enzyme responsible for converting $\mathrm{Fe}^{3+}$ to $\mathrm{Fe}^{2+}$, blocked erastin-induced or RSL3-induced cell death and HMGB1 release in Nupr $1^{-/}$mPDAC cells (Fig. 2f-h). Together, these findings indicate that NUPR1 blocks ferroptosis through the inhibition of iron-dependent oxidative damage.

LCN2 acts as an effector gene of NUPR1 in blocking ferroptosis. The levels of intracellular iron are determined by its uptake, storage, release, and metabolism ${ }^{25}$. In brief, after uptake by transferrin receptor (TFRC, also known as TFR1), $\mathrm{Fe}^{3+}$ is reduced to $\mathrm{Fe}^{2+}$ by STEAP3 and then released from the endosome to the cytoplasm by solute carrier family 11 member 2 (SLC11A2, also known as DMT1). Ferritin, including ferritin light chain (FTL) and ferritin heavy chain 1 (FTH1), functions as a major iron storage protein. Finally, the release of $\mathrm{Fe}^{2+}$ into the extracellular space requires iron transporters, such as solute carrier family 40 member 1 (SLC40A1, also known as ferroportin-1) and yet another ion transporter, LCN2. Among the key genes for iron metabolism (e.g., Lcn2, Tfrc, Steap3, Slc11a2, Ftl, Fth1, and Slc40a1), erastin-induced or RSL3-induced the upregulation of Lcn2 was completely blocked in Nupr1-/- mPDAC 


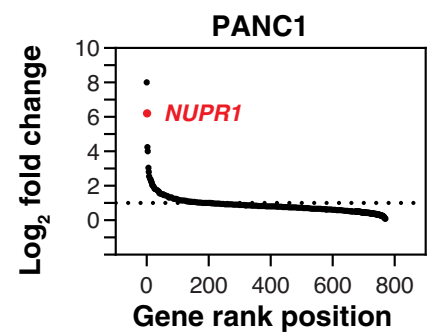

BxPC3

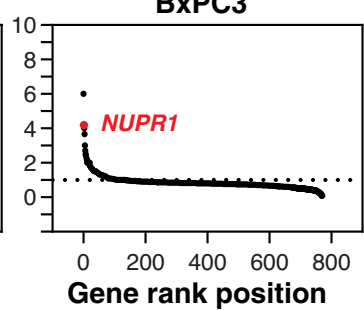

b

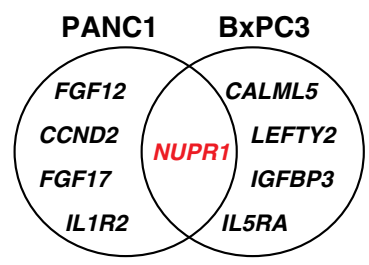

Top 5 up-regulated genes

C

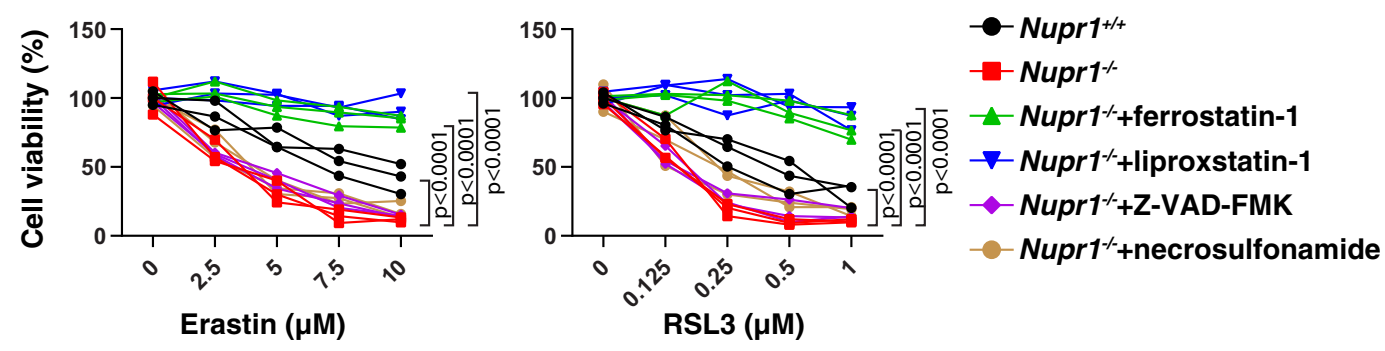

d
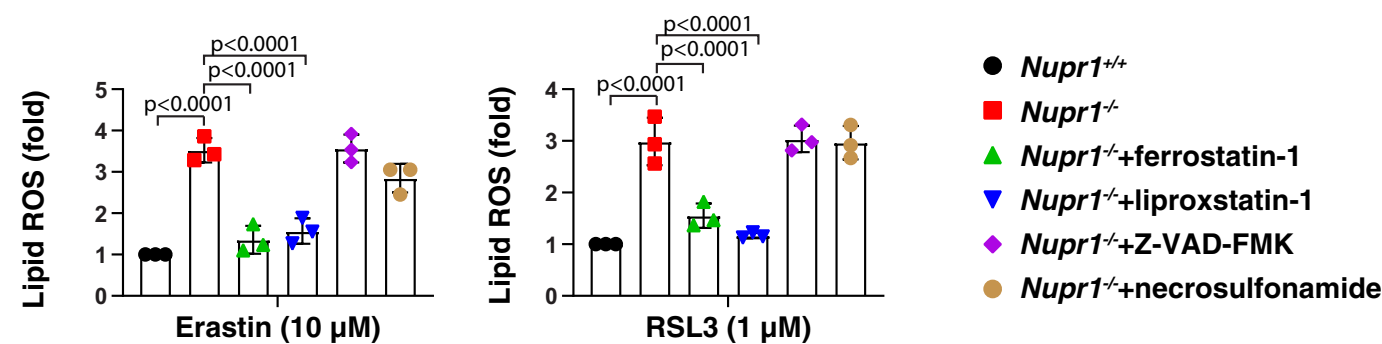

RSL3 $(1 \mu \mathrm{M})$

e

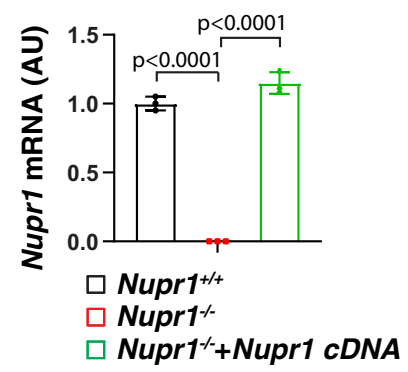

f

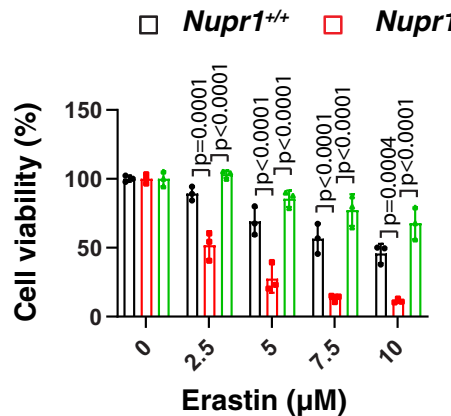

Nupr1\%+Nupr1 cDNA

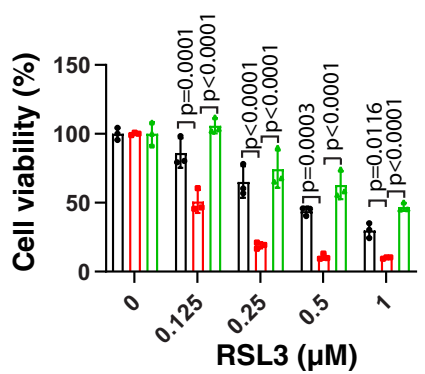

Fig. 1 NUPR1 acts as a repressor of ferroptosis. a A NanoString technology-based screening of differentially expressed tumor-associated genes in PANC1 and BxPC3 cells following treatment with erastin $(10 \mu \mathrm{M})$ for $24 \mathrm{~h}$. b Top 5 upregulated genes. c, d Nupr $1^{+/+}$and Nupr1-/- mPDAC cells were treated with erastin or RSL3 in the absence or presence of ferrostatin-1 $(1 \mu \mathrm{M})$, liproxstatin-1 $(1 \mu \mathrm{M})$, Z-VAD-FMK $(10 \mu \mathrm{M})$, or necrosulfonamide $(1 \mu \mathrm{M})$ for $24 \mathrm{~h}$, and then cell viability (c) and lipid ROS (d) was measured ( $n=3$ well/group, two-way ANOVA with Tukey's multiple comparisons test on all pairwise combinations). e qPCR analysis of NUPR1 expression in indicated mPDAC cells ( $n=3$ well/group, one-way ANOVA with Tukey's multiple comparisons test on all pairwise combinations). $\mathbf{f}$ Cell viability of indicated mPDAC cells following treatment with erastin or RSL3 for $24 \mathrm{~h}$ ( $n=3$ well/group, two-way ANOVA with Tukey's multiple comparisons test on all pairwise combinations). Data in $\mathbf{d}-\mathbf{f}$ are presented as mean \pm SD. The results in c-f are representative of those from 2 to 3 independent experiments with three technical replicates each. The results in a are representative of those from one independent experiment with three technical replicates each.

cells (Fig. 3a). Consistent with the mRNA assay, erastin-induced or RSL3-induced protein expression of LCN2 was abolished in Nupr $1^{-/}$mPDAC cells (Fig. 3b). Luciferase reporter gene (Fig. 3c) and chromatin immunoprecipitation (Fig. 3d) assays further revealed that $L c n 2$ is a direct target gene of NUPR1 in mPDAC cells during ferroptosis. As expected, the knockdown of ATF4 by shRNA suppressed LCN2 mRNA expression in PANC1 cells following erastin or RSL3 treatment (Supplementary Fig. 1e). However, overexpression of ATF4 failed to induce Lcn2 upregulation in Nupr $1^{-/-}$mPDAC cells following erastin or RSL3 treatment (Supplementary Fig. 1f, g). These findings confirm that ATF4-dependent NUPR1 expression is required for subsequent LCN2 expression during ferroptosis.

We next examined whether the genetic silence of LCN2 has a pro-ferroptotic phenotype similar to that of Nupr1-deficient cells. Indeed, shRNA-based Lcn2/LCN2 suppression increased $\mathrm{Fe}^{2+}$ accumulation, oxidative damage (MDA and 8-OHdG), HMGB1 release and cell death in mPDAC (Fig. 3e-j) or PANC1 
a

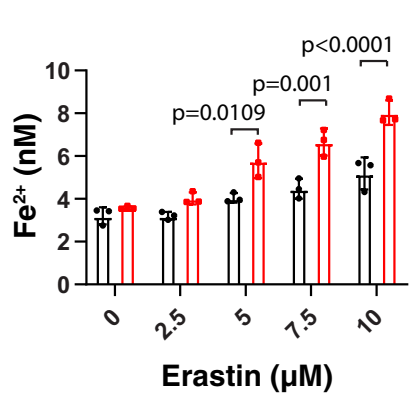

Erastin $(\mu \mathrm{M})$

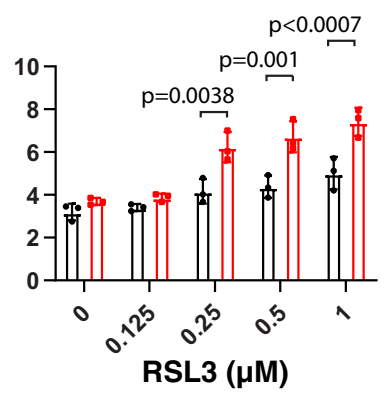

d

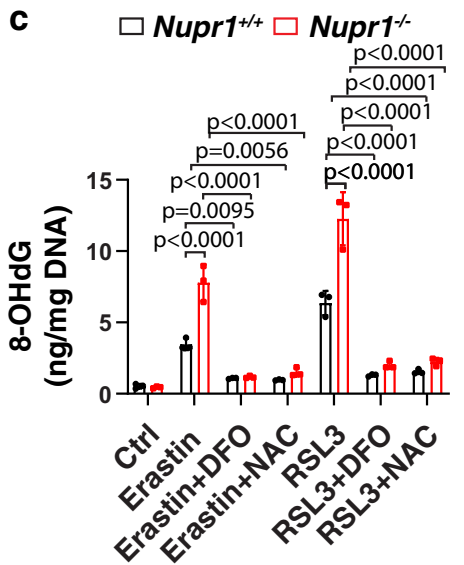

f $\square \mathrm{Nupr}^{1+/} \square \mathrm{Nupr}^{1 /-}$

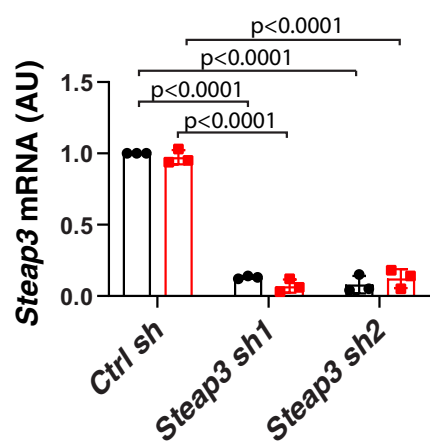

g b

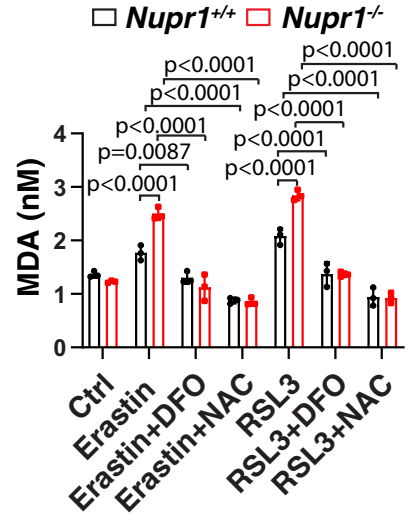

e $\square$ Nupr1 $^{+/+} \square$ Nupr1 $^{-/}$

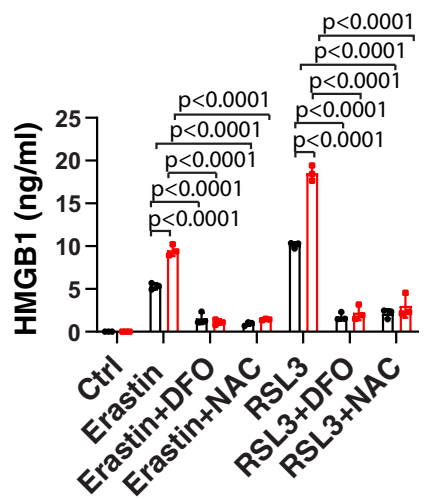

$\square$ Nupr1 $^{+/+} \square$ Nupr1 $^{-1}$

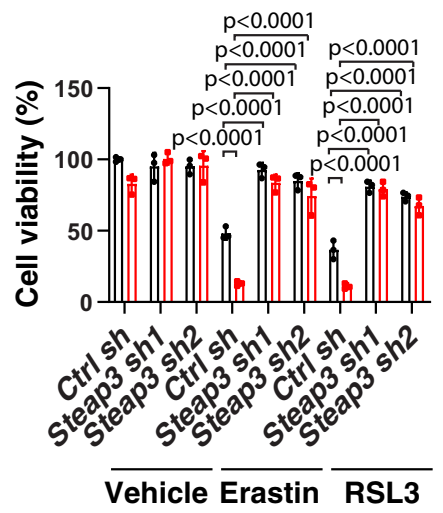

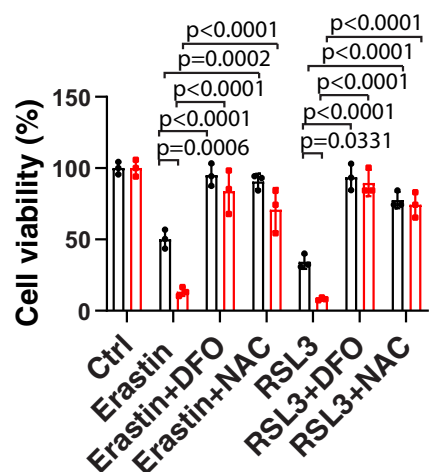

h

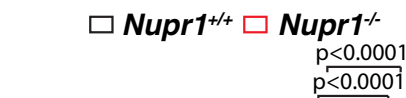
$\mathrm{p}<0.0001$
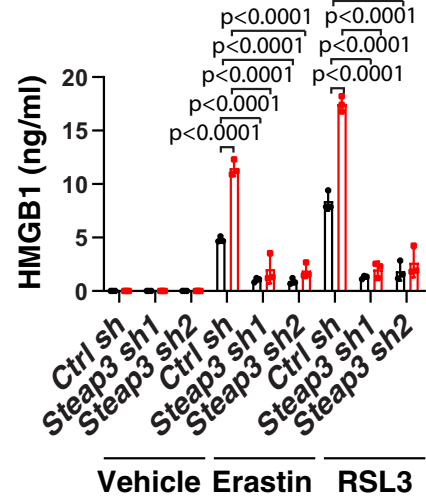

Fig. 2 NUPR1 inhibits iron-dependent oxidative damage in ferroptosis. a Fe $\mathrm{e}^{2+}$ levels in indicated mPDAC cells following treatment with erastin or RSL3 for $24 \mathrm{~h}$ ( $n=3$ well/group, two-way ANOVA with Tukey's multiple comparisons test on all pairwise combinations). b-e Indicated mPDAC cells were treated with erastin $(10 \mu \mathrm{M})$ or RSL3 $(1 \mu \mathrm{M})$ in the absence or presence of DFO $(100 \mu \mathrm{M})$ or NAC $(1 \mathrm{mM})$ for $24 \mathrm{~h}$, and then intracellular MDA (b), intracellular 8-OHdG (c), extracellular HMGB1 (d), and cell viability (e) were assayed ( $n=3$ well/group, two-way ANOVA with Tukey's multiple comparisons test on all pairwise combinations). $\mathbf{f}$ qPCR analysis of Steap3 mRNA in indicated mPDAC cells ( $n=3$ well/group, two-way ANOVA with Tukey's multiple comparisons test on all pairwise combinations). $\mathbf{g}$, h Indicated mPDAC cells were treated with erastin (10 $\mu \mathrm{M})$ or RSL3 (1 $\mu \mathrm{M})$ or vehicle ( $0.01 \%$ dimethyl sulfoxide) for $24 \mathrm{~h}$, and then extracellular HMGB1 (g) and cell viability (h) were determined ( $n=3$ well/group, two-way ANOVA with Tukey's multiple comparisons test on all pairwise combinations). Data in $\mathbf{a}-\mathbf{h}$ are presented as mean \pm SD. The results in $\mathbf{a}-\mathbf{h}$ are representative of those from 2 to 3 independent experiments with three technical replicates each.

(Supplementary Fig. 4) cells following treatment with erastin or RSL3, which was reversed by DFO or the ferroptosis inhibitor liproxstatin-1. These findings indicate that LCN2 plays a similar role as NUPR1 in the inhibition of ferroptosis.

To determine whether the downregulation of LCN2 is essential for the induction of ferroptosis, we re-expressed Lcn2 in Nupr1-/-
mPDAC cells by transfecting the $L n c 2$ gene (Fig. 4a). The transfection enforced expression of $L c n 2$ restored ferroptosis resistance in $N u p r 1^{-/-}$mPDAC cells, which was associated with decreased $\mathrm{Fe}^{2+}$ accumulation, oxidative damage (MDA and $8-\mathrm{OHdG}$ ), HMGB1 release, and cell death (Fig. $4 \mathrm{~b}-\mathrm{f}$ ). Thus, our findings demonstrated that NUPR1 blocks ferroptotic cell 
a

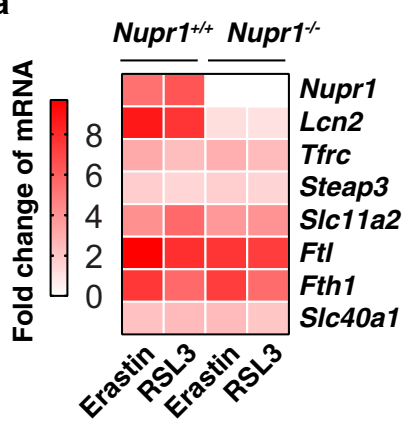

b

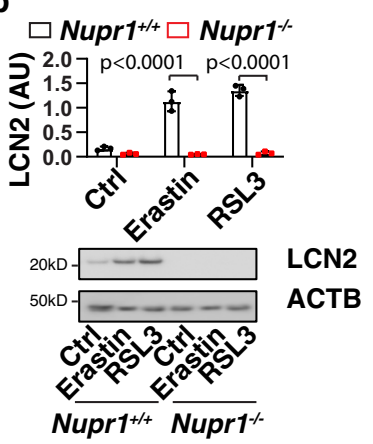

C

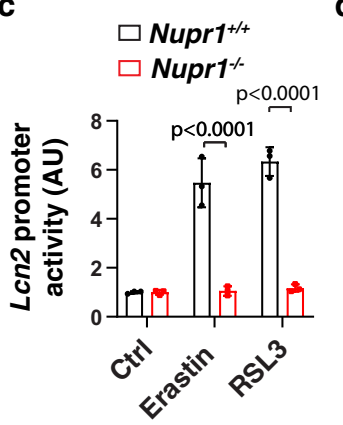

d

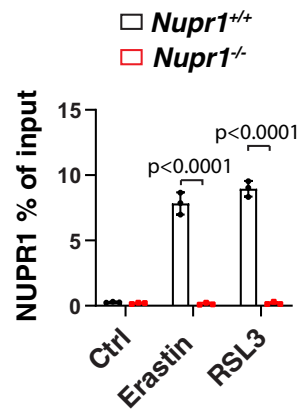

e

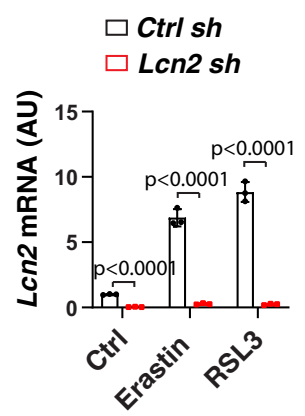

f

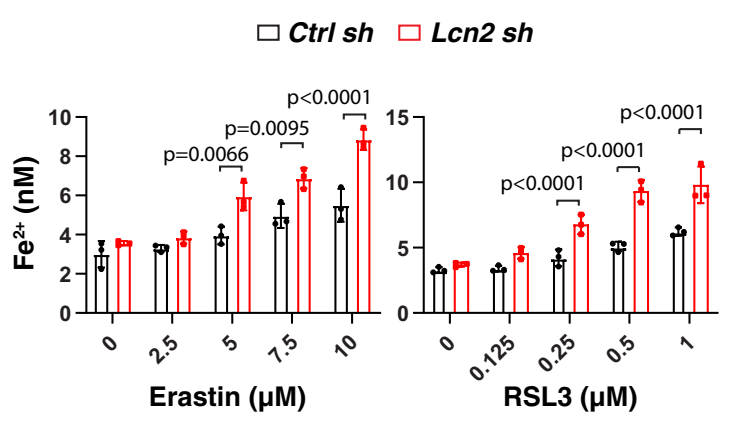

g

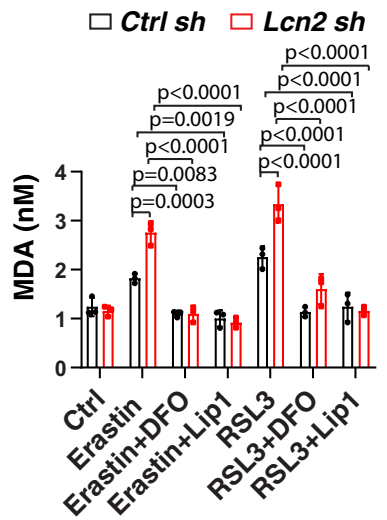

h

i

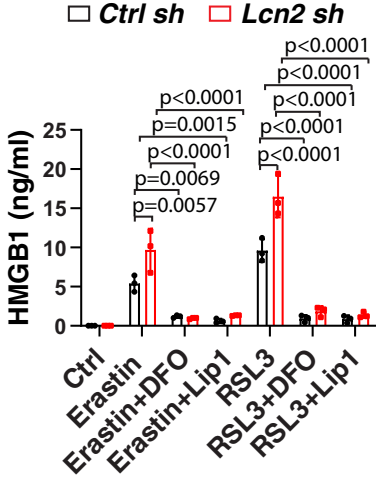

j

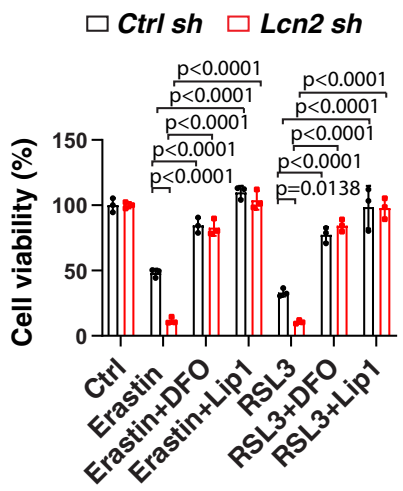

Fig. 3 LCN2 acts as an effector gene of NUPR1 in blocking ferroptosis. a Heatmap of relative mRNA levels of iron metabolism-associated genes in Nupr $1^{+/+}$and Nupr1-/- mPDAC cells following treatment with erastin $(10 \mu \mathrm{M})$ or RSL3 $(1 \mu \mathrm{M})$ for $24 \mathrm{~h}$. b, c Analysis of LCN2 protein expression and Lcn2 promoter activity in Nupr1 $1^{+/+}$and Nupr1 $1^{-/-}$mPDAC cells following treatment with erastin $(10 \mu \mathrm{M})$ or RSL3 $(1 \mu \mathrm{M})$ for $24 \mathrm{~h}(n=3$ well/group, one-tailed $t$ test). d Binding of NUPR1 to Lcn2 promoter was analyzed using ChIP-qPCR in indicated mPDAC cells following treatment with erastin (10 $\mu M$ ) or RSL3 $(1 \mu \mathrm{M})$ for $24 \mathrm{~h}$ ( $n=3$ well/group, one-tailed $t$ test). e qPCR analysis of $L c n 2 \mathrm{mRNA}$ in indicated mPDAC cells following treatment with erastin (10 $\mu \mathrm{M})$ or $\operatorname{RSL3}(1 \mu \mathrm{M})$ for $24 \mathrm{~h}$ ( $n=3$ well/group, one-tailed $t$ test). $\mathbf{f ~ F e}{ }^{2+}$ levels in indicated mPDAC cells following treatment with erastin or RSL3 for $24 \mathrm{~h}(n=3$ well/group, two-way ANOVA with Tukey's multiple comparisons test on all pairwise combinations). $\mathbf{g}-\mathbf{j}$ Indicated mPDAC cells were treated with erastin $(10 \mu \mathrm{M})$ or RSL3 $(1 \mu \mathrm{M})$ in the absence or presence of DFO $(100 \mu \mathrm{M})$ or liproxstatin-1 $(1 \mu \mathrm{M})$ for $24 \mathrm{~h}$, and then intracellular MDA (g), intracellular $8-O H d G$ (h), extracellular HMGB1 (i), and cell viability (j) were quantified ( $n=3$ well/group, two-way ANOVA with Tukey's multiple comparisons test on all pairwise combinations). Data in $\mathbf{b}-\mathbf{j}$ are presented as mean \pm SD. The results in $\mathbf{a}-\mathbf{j}$ are representative of those from 2 to 3 independent experiments with three technical replicates each.

death through inducing the expression of the iron transporter LNC2.

The NUPR1-LCN2 pathway limits the anticancer activity of IKE in vivo. We next sought to determine whether the inhibition of the NUPR1-LCN2 pathway can enhance the in vivo anticancer activity of imidazole ketone erastin (IKE), a metabolically stable analog of erastin ${ }^{26}$. Consistent with our in vitro observations, NUPR1-knockdown (NUPR1 $\left.{ }^{K D}\right)$ or LCN2-knockdown $\left(L C N 21^{K D}\right)$
PANC1 cells were more sensitive to IKE-induced tumor suppression compared to control groups in vivo, in a model in which immunodeficient mice were bearing human PDAC cells (Fig. 5a). The inhibition of the NUPR1-LCN2 pathway conferred therapy sensitivity that was associated with increased intratumoral $\mathrm{Fe}^{2+}$ (Fig. 5b) or MDA levels (Fig. 5c), mRNA expression of prostaglandin-endoperoxide synthase 2 (PTGS2, an inducible enzyme associated with inflammation and cell death events, including ferroptosis ${ }^{27}$ ) (Fig. 5d) and circulating HMGB1 protein 
a

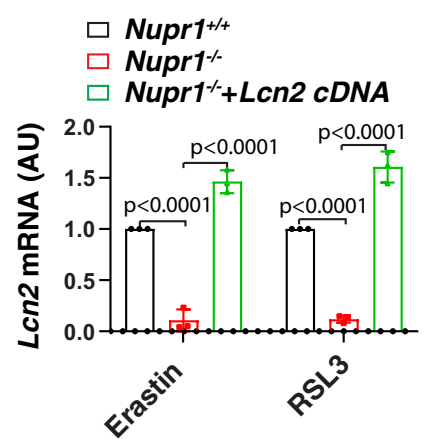

c

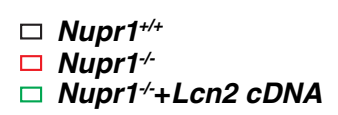

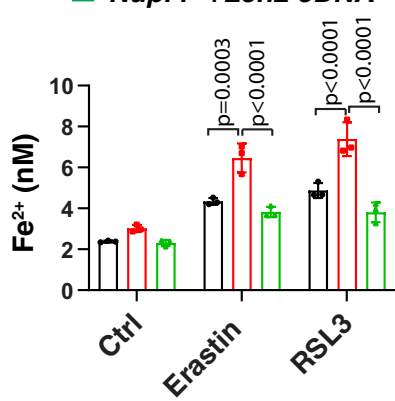

f

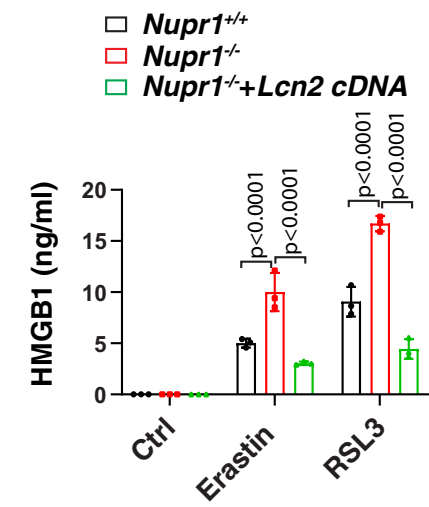

b

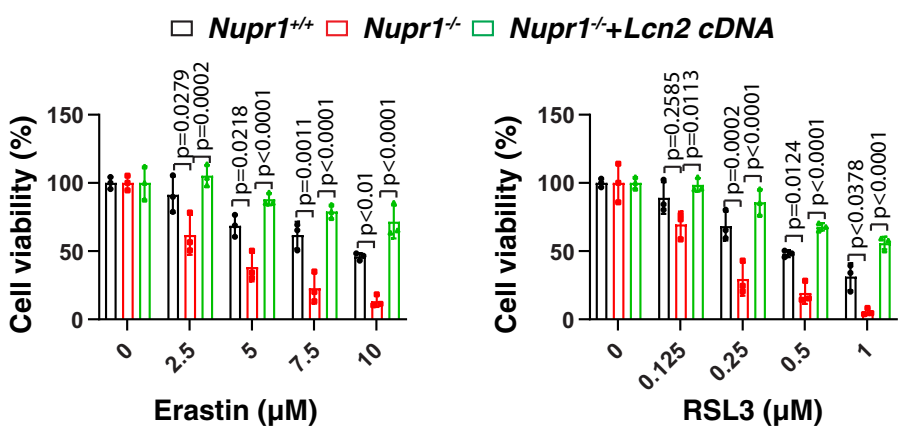

d

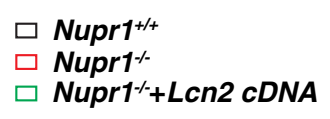

$\mathbf{e}$

\section{$\square$ Nupr1 $^{+/+}$ \\ $\square$ Nupr1 ${ }^{-1}$ \\ $\square$ Nupr1 ${ }^{-+L c n 2 ~ c D N A ~}$}
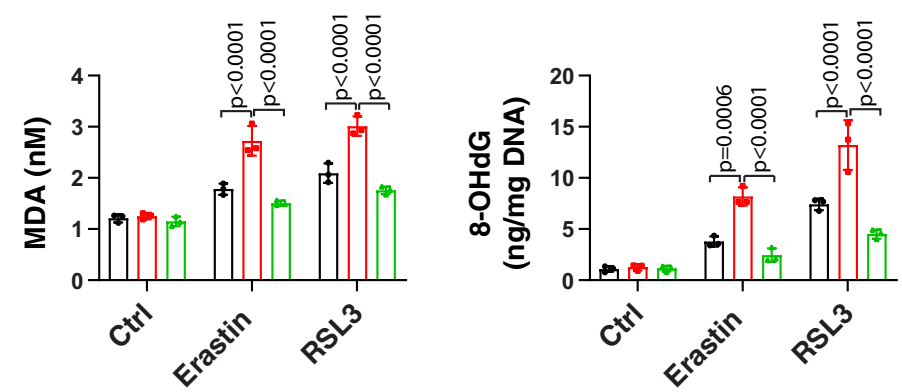

Fig. 4 Re-expression of LCN2 rescues ferroptosis resistance in Nupr1-deficient cells. a qPCR analysis of $L$ cn2 mRNA in indicated mPDAC cells following treatment with erastin $(10 \mu \mathrm{M})$ or RSL3 $(1 \mu \mathrm{M})$ for $24 \mathrm{~h}$ ( $n=3$ well/group, two-way ANOVA with Tukey's multiple comparisons test on all pairwise combinations). b Cell viability in indicated mPDAC cells following treatment with erastin or RSL3 for $24 \mathrm{~h}$ ( $n=3$ well/group, two-way ANOVA with Tukey's multiple comparisons test on all pairwise combinations). c-f Indicated mPDAC cells were treated with erastin (10 $\mu \mathrm{M})$ or RSL3 (1 $\mu \mathrm{M})$ for $24 \mathrm{~h}$, and then intracellular Fe ${ }^{2+}(\mathbf{c})$, intracellular MDA (d), intracellular 8-OHdG (e), and extracellular HMGB1 were assayed ( $n=3$ well/group, two-way ANOVA with Tukey's multiple comparisons test on all pairwise combinations). Data in a-f are presented as mean \pm SD. The results in a-f are representative of those from 2 to 3 independent experiments with three technical replicates each.

(Fig. 5e), all of which were inhibited by the ferroptosis inhibitor liproxstatin-1. In contrast, the apoptosis inhibitor Z-VAD-FMK or the necroptosis inhibitor necrosulfonamide had no effects on the increased anticancer activity of IKE in NUPR1 $1^{K D}$ or $L C N 21^{K D}$ PANC1 cells (Supplementary Fig. 5). ZZW-115, a potent NUPR1 inhibitor ${ }^{28}$, also increased the anticancer activity of IKE in PANC1 or MIAPaCa2 xenograft mouse models (Fig. 5f). These animal studies support the contention that the NUPR1-LCN2 pathway limits the anticancer activity of IKE. The synergistic effect on cell death by ZZW-115 and erastin or RSL3 was diminished in Nupr1 ${ }^{-1}$ cells, but not in Nupr1 ${ }^{+/+}$cells (Fig. 5g), indicating that ZZW-115 bound NUPR1 possesses dominant negative activity or, in other words, the inhibition of NUPR1 is more effective than the loss of NUPR1.
LCN2 prevents pancreatitis. Excess iron is stored in multiple organs, including the pancreas, which may cause tissue injury and inflammation under pathologic conditions ${ }^{29}$. We further examined the effects of $L \mathrm{cn} 2$ depletion-mediated iron accumulation on L-arginine-induced acute pancreatitis in mice, a widely used experimental model that can cause pancreatic oxidative injury, sterile inflammation, and extensive necrosis ${ }^{30}$. We generated pancreas-specific Lcn2-knockout mice (Pdx1-Cre; Lcn $2^{f l o x} / f l o x$, termed Lcn2Pan-/- mice) by crossing Lcn2flox/flox and Pdx1-Cre mice. Western blot confirmed the expression of LCN2 was diminished in the pancreas (but not in the liver) from $\mathrm{LCn}_{2} \mathrm{Pan}-1-$

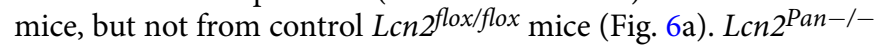
mice were more sensitive to L-arginine-induced acute pancreatitis, 
a

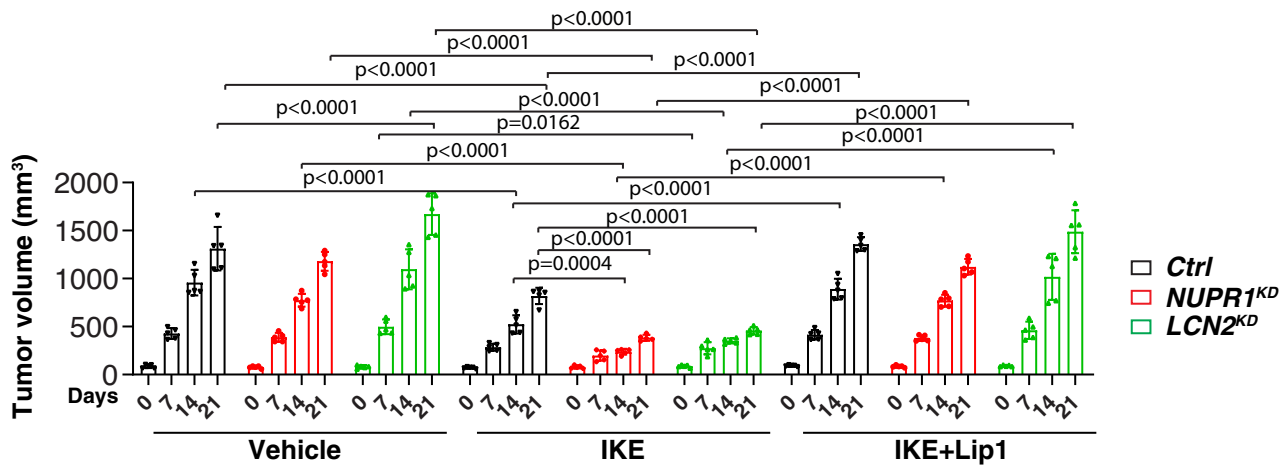

b

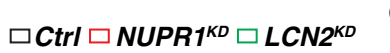

C

$\square$ CtrI $\square$ NUPR1 ${ }^{K D} \square L C N 2^{K D}$ d

d $\quad \square$ CtrI $\square N U P R 1^{K D} \square L C N 2^{K D}$
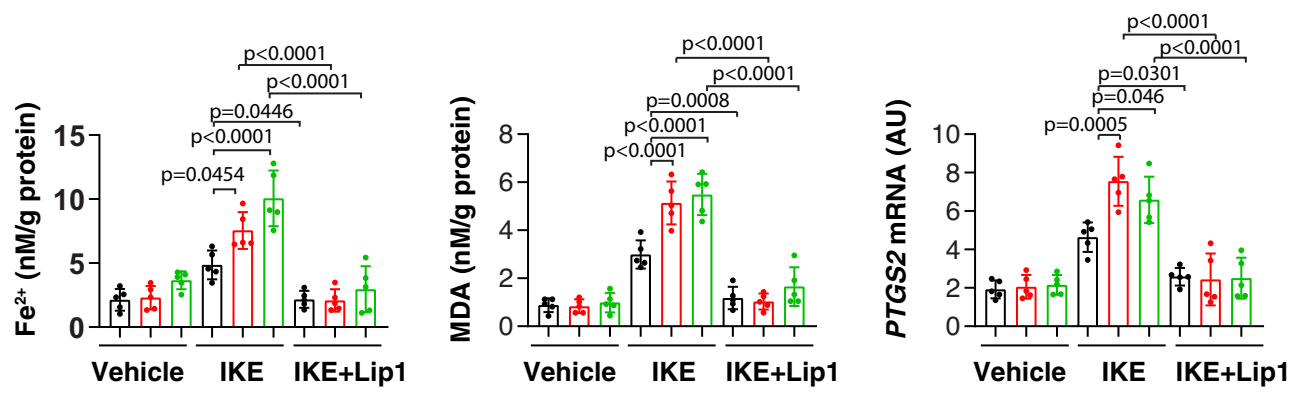

e

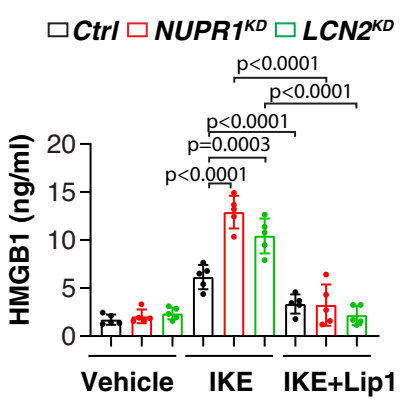

f
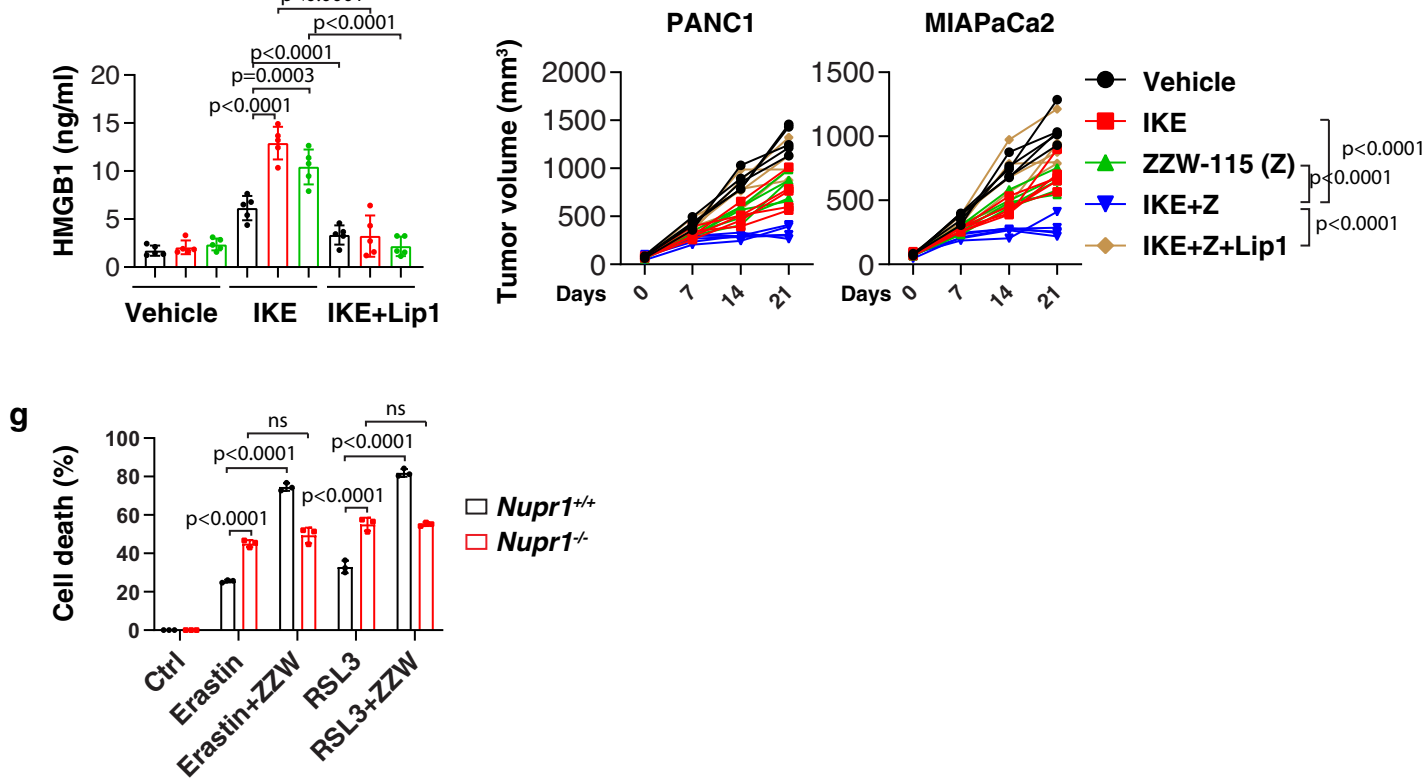

Fig. 5 The NUPR1-LCN2 pathway limits ferroptotic cancer cell death in vivo. a Athymic nude mice were injected subcutaneously with indicated PDHA1knockdown (NUPRT KD) or $L C N 2-k n o c k d o w n\left(L C N 2^{K D}\right)$ PANC1 cells for 7 days and then treated with IKE (40 mg/kg, i.p., once every other day) in the absence or presence of liproxstatin-1 (10 mg/kg, i.p., once every other day) at day 7 for 2 weeks. Tumor volumes were calculated weekly ( $n=5$ mice/ group; two way ANOVA with Tukey's multiple comparisons test on all pairwise combinations). b-e In parallel, the levels of Fe ${ }^{2+}$ (b), MDA (c), or PTGS2 mRNA (d) in isolated tumors and serum HMGB1 (e) at day 14 after treatment were assayed ( $n=5$ mice/group; one-way ANOVA with Tukey's multiple comparisons test on all pairwise combinations). f Athymic nude mice were injected subcutaneously with PANC1 or MIAPaCa2 cells for 7 days and then treated with IKE (40 mg/kg, i.p., once every other day) in the absence or presence of ZZW-115 (5 mg/kg, i.p., once every other day) or liproxstatin-1 $(10 \mathrm{mg} / \mathrm{kg}$, i.p., once every other day) at day 7 for 2 weeks. Tumor volumes were calculated weekly ( $n=5$ mice/group; two-way ANOVA with Tukey's multiple comparisons test on all pairwise combinations). $\mathbf{g}$ Indicated mPDAC cells were treated with erastin (10 $\mu$ M) or RSL3 (1 $\mu$ M) in the absence or presence ZZW-115 $(2 \mu \mathrm{M})$ for $24 \mathrm{~h}$ and then cell death was assayed ( $n=3$ well/group, two-way ANOVA with Tukey's multiple comparisons test on all pairwise combinations). ns: not significant. Data in $\mathbf{a}-\mathbf{e}$ and $\mathbf{g}$ are presented as mean \pm SD. The results in $\mathbf{a}-\mathbf{g}$ are representative of those from 2 to 3 independent experiments. 
a
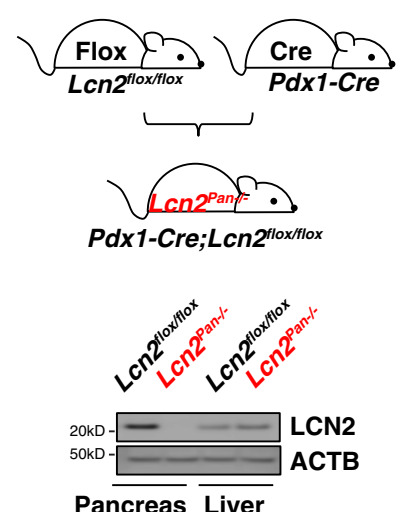

Pancreas Liver b

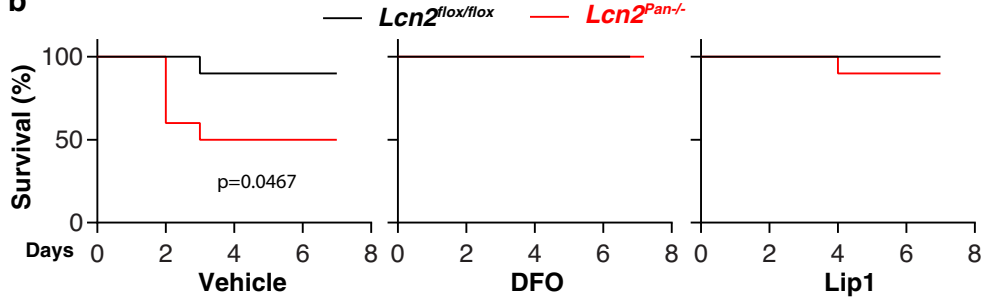

c

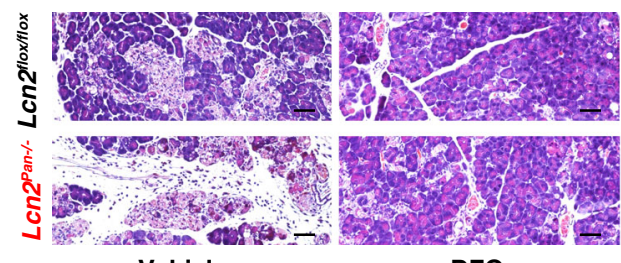

DFO

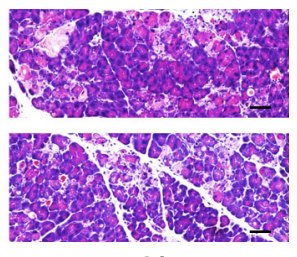

Lip1 d

d

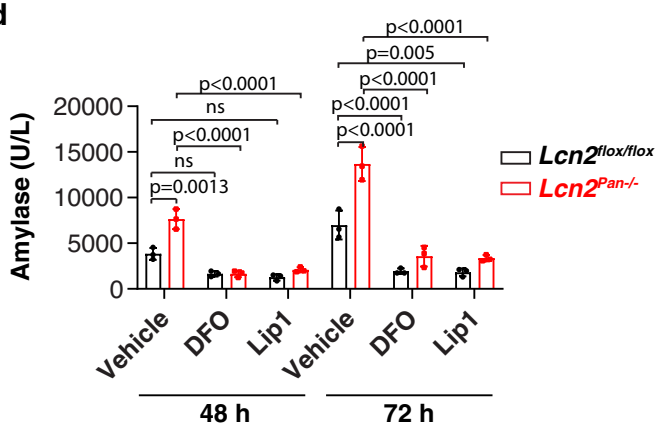

f

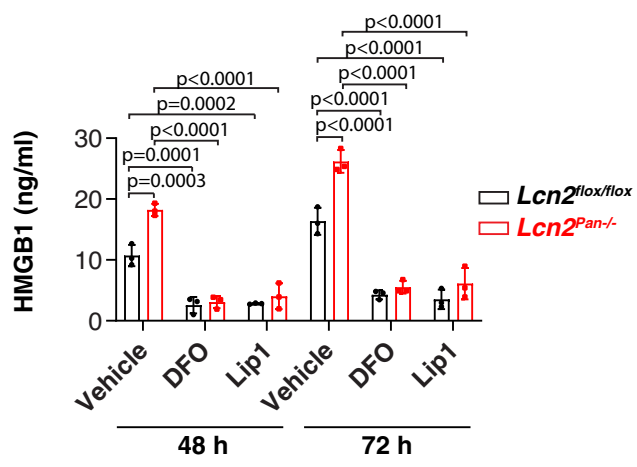

h

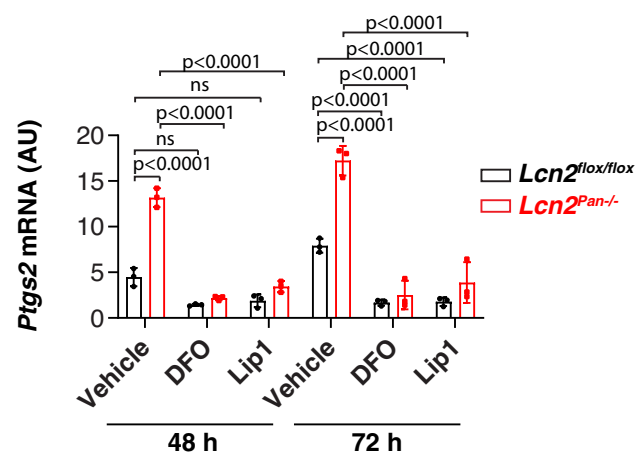

e

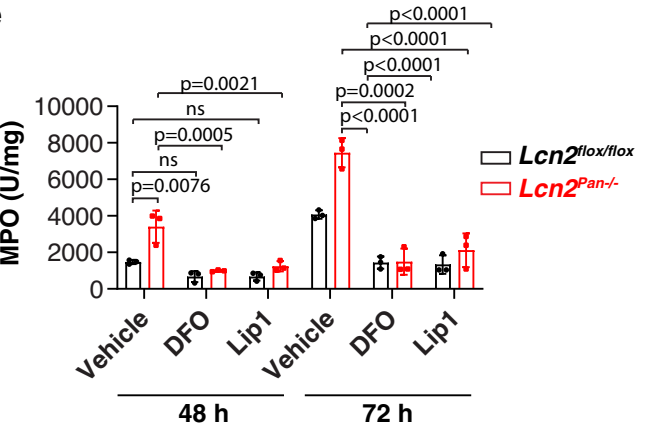

9

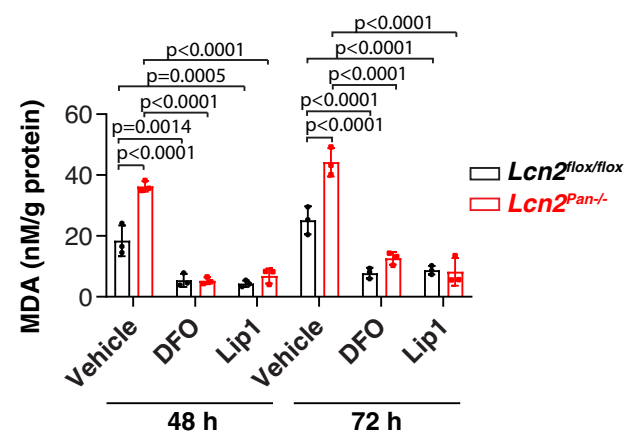

Fig. 6 LCN2 prevents pancreatitis through the inhibition of ferroptosis. a Western blot analysis of LCN2 expression in the pancreas and liver in

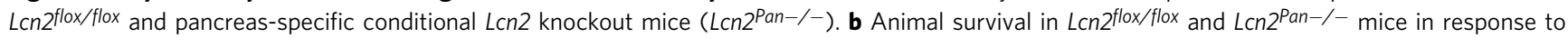
L-arginine-induced acute severe pancreatitis in the absence or presence of liproxstatin-1 or DFO ( $n=10$ mice/group; one-sided Log-rank [Mantel-Cox] test). c-h In parallel, hematoxylin and eosin-stained pancreatic sections (c), serum amylase (d), pancreatic MPO (e), serum HMGB1 (f), pancreatic MDA $(\mathbf{g})$, and mRNA expression of Ptgs2 in the pancreas $(\mathbf{h})$ were assayed at the indicated time points ( $n=3$ mice/group; two-way ANOVA with Tukey's multiple comparisons test on all pairwise combinations). Bar $=50 \mu \mathrm{m}$. ns: not significant. Data in $\mathbf{d}-\mathbf{h}$ are presented as mean \pm SD. The results in $\mathbf{b}-\mathbf{h}$ are representative of those from two independent experiments. The results in (a) are from an independent experiment. 


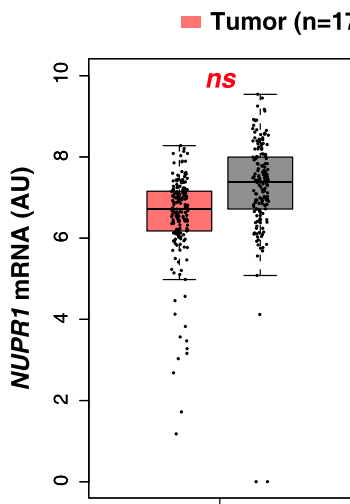

Normal $(n=171)$

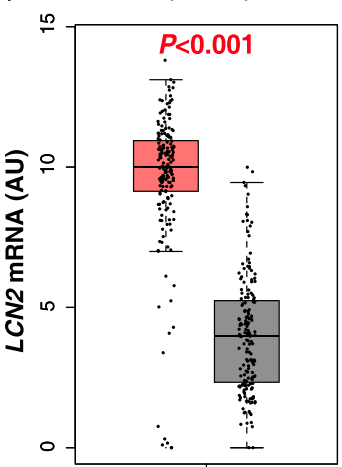

c

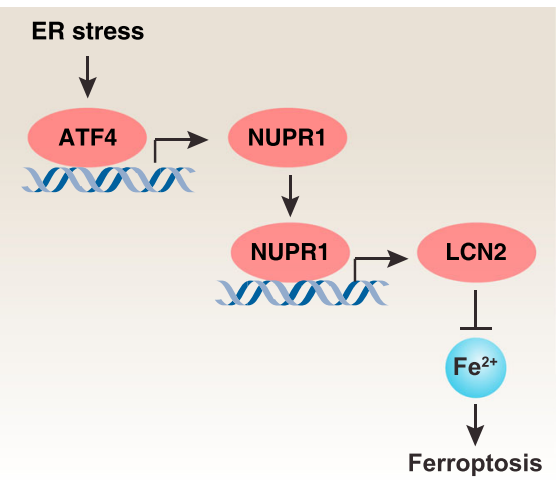

b

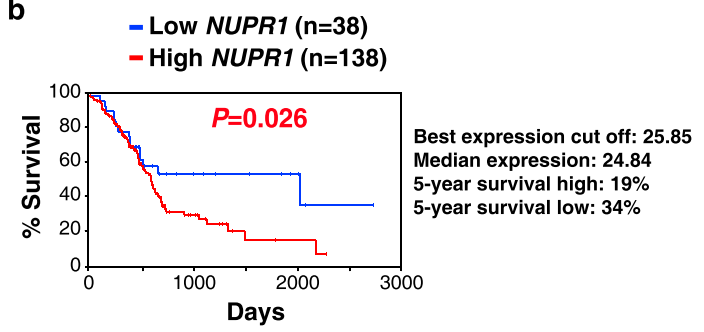

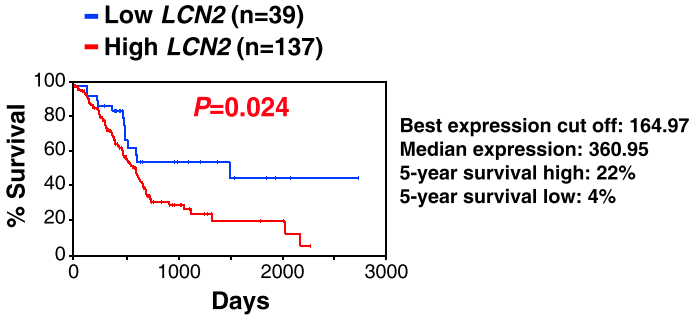

Fig. 7 Prognostic significance of the NUPR1-LCN2 pathway in human PDAC. a Upregulation of LCN2 (but not NUPR1) gene expression within the tumor from PDAC patients compared to normal controls using datasets from the Cancer Genome Atlas (TCGA) (one-tailed $t$ test). The data are presented as box-and-whisker plots. Boxes represent the median and the 25th and 75th percentiles. ns: not significant. $n$ is number of cases. $\mathbf{b}$ Kaplan-Meier survival analysis of NUPR1 and LCN2 gene expression in PDAC patients using TCGA datasets (one-sided Log-rank [Mantel-Cox] test). $n$ is number of cases. c Schematic representation of the role of NUPR1 in the regulation of ferroptosis in human PDAC cells.

hence exhibiting increased mortality (Fig. 6b), aggravated pancreatic histological damage (Fig. 6c), and elevated serum amylase (a diagnostic biomarker of acute pancreatitis) (Fig. 6d), pancreatic myeloperoxidase (MPO, a marker of neutrophil recruitment) (Fig. 6e), serum HMGB1 (Fig. 6f), pancreatic MDA (Fig. 6g), and Ptgs 2 mRNA (Fig. 6h). This phenotype of Lcn2 depletionmediated acute pancreatitis could be prevented by treatment with the ferroptosis inhibitor liproxstatin-1 or the iron chelator DFO (Fig. 6b-h). Collectively, these studies suggest that LCN2 exerts a protective effect on acute pancreatitis potentially through the inhibition of ferroptotic response.

Prognostic significance of the NUPR1-LCN2 pathway in human PDAC. Previous animal studies indicate that NUPR1 and LCN2 may play an oncogene-like role in PDAC ${ }^{31,32}$. To test this possibility, we carried out bioinformatics analyses using a publicly available gene expression dataset: the Cancer Genome Atlas (TCGA). LCN2 (but not NUPR1) mRNA expression was upregulated in the PDAC tumor group compared to the normal group (Fig. 7a). An overall survival assay revealed that a low expression of NUPR1 or LCN2 was correlated with increased survival of PDAC patients (Fig. 7b). These analyses indicate that activation of the NUPR1-LCN2 pathway may contribute to the development of human PDAC.

\section{Discussion}

There is a clear role for transcription factors in human health and disease, highlighting the importance of continued efforts for understanding gene expression modulation in physiological and pathological processes, including cell death ${ }^{33}$. In this study, we demonstrated that NUPR1 is critical for activating a transcriptional program that may block ferroptotic cell death or tissue injury through the upregulation of LCN2 expression (Fig. 7c), providing a potential strategy for the treatment of ferroptosisrelated diseases.

NUPR1 is a member of AT hook-containing chromosomal DNA-binding proteins that was first identified and cloned in a study of pancreatitis-induced tissue injury ${ }^{34}$. Like other architectural chromatin-binding proteins, NUPR1 participates in a wide range of DNA-relevant events, such as gene transcription, DNA repair, and chromosome recombination ${ }^{35-39}$. NUPR1 is also a multifunctional stress-inducible protein that is produced in response to diverse environmental stresses, including oxidative damage and the unfolded protein response ${ }^{40-45}$. ATF4, a repressor of ferroptosis in PDAC cells ${ }^{22}$, has been shown to regulate NUPR1 expression in response to various stresses, including ER stress ${ }^{46}$. We found that ATF4 is required for NUPR1 expression in response to ferroptosis activators. NUPR1 regulates cellular processes mainly by activating its target genes $^{40,47}$. Our current study establishes an inhibitory role for NUPR1 in regulating ferroptosis through directly upregulating LCN2 expression. We found that the depletion of NUPR1 or LCN2 had a similar pro-ferroptotic phenotype in human or mouse PDACs, whereas re-expression of LCN2 reversed the exaggerated ferroptosis observed in $N u p r 1^{-1-}$ cells. Combined with other studies on models of apoptosis ${ }^{31,48}$, necroptosis ${ }^{28,44}$ and autophagy-dependent cell death ${ }^{41,49,50}$, the present study suggests that the activation of NUPR1 may play a universal cytoprotective role. Since ATF4 has been shown to promote the expression of solute carrier family 7 member 11 (SLC7A11, a component of the antiporter system $\mathrm{xc}^{-}$that inhibits ferroptosis by importing the amino acid cystine for glutathione production in cells $)^{21}$, it is necessary to investigate whether NUPR1 is also involved in ATF4-mediated SLC7A11 expression in the future. 
Our studies reveal a role for NUPR1 in preventing intracellular iron accumulation in response to ferroptosis activators. Ferroptosis is generally recognized as an iron-dependent oxidative death, which can be suppressed by iron chelators, the depletion of the iron uptake receptor TFRC or the overexpression of the iron storage protein ferritin ${ }^{8}$. Given that depletion of NUPR1 increased iron accumulation induced by classical ferroptosis activators (erastin or RSL3), the expression levels of core iron metabolismassociated genes were measured in WT and Nupr1-l- cells. Such analyses led to the conclusion that $L c n 2$, but none of the other iron metabolism-relevant genes, functions as a direct downstream target gene regulated by NUPR1. Although the function of LCN2 in iron metabolism is still unclear, it has been suggested to be involved in the extrusion of iron from the intracellular to the extracellular compartment ${ }^{51-53}$. Blocking NUPR1-dependent LCN2 expression significantly increased intracellular iron concentrations and subsequent oxidative damage, including lipid peroxidation and DNA damage. These observations established a NUPR1-dependent LCN2-regulatory pathway for ferroptosis (Fig. 7c).

While the function of mitochondria in ferroptosis is contextdependent ${ }^{3,54,55}$, mitochondria are widely recognized as a major source of iron-induced $\operatorname{ROS}^{56}$. Mitochondria also utilize iron for the synthesis of heme and iron sulfur, which can form a feedback to amplify or diminish ferroptosis ${ }^{57,58}$. In view of previously established links between the downregulation of NUPR1 expression and the increased mitochondria-dependent apoptosis in some types of cells $s^{31,48}$, it is possible that NUPR1 downregulation-mediated iron accumulation functions as a common upstream signal to trigger mitochondrial dysfunction, thus compromising cellular survival and fitness by negatively affecting aerobic glycolysis, oxidative phosphorylation, and fatty acid synthesis $44,54,59-62$.

Our findings highlight a potential functional role of the NUPR1-LCN2 pathway in the regulation of ferroptosis-related tumor therapy. While the deletion of Nupr 1 in mice prevents mutated Kras-induced tumorigenesis ${ }^{31,63}$, we found that the pharmacological or genetic inhibition of the NUPR1-LCN2 pathway enhanced anticancer activity of ferroptosis activators in PDAC cells in vitro or in preclinical mouse models. Ferroptosis seems to play a dual role in tumor biology, which is contextdependent. On the one hand, ferroptosis activators can kill cancer cells or active antitumor immunity to suppress tumor growth ${ }^{64-66}$. On the other hand, ferroptosis-mediated inflammatory response may promote tumor growth through the release of DAMPs ${ }^{67,68}$. Further exploration of the cellular and functional relevance of ferroptosis in the tumor microenvironment would be important to develop better anticancer therapeutic strategies.

We addressed a potential pathologic link between ferroptosis and pancreatitis using $\operatorname{Lcn} 2^{P a n-/-}$ mice, knowing that excessive ferroptosis can compromise the function of entire organs including the kidney, liver, brain, heart, and pancreas ${ }^{7}$. Compared to WT mice, $L c n 2^{P a n-/-}$ mice exhibited increased sensitivity to experimental pancreatitis-mediated animal death. In contrast, the inhibition of ferroptosis by liproxstatin-1 or by using the iron chelator DFO protected against pancreatic injury, lipid peroxidation, HMGB1 release, and PTGS2 expression, leading to prolonged animal survival in $L c n 2^{P a n-/-}$ mice. In addition to PTGS2, transferrin receptor (TFRC) and acyl-CoA synthetase long chain family member 4 (ACSL4) have also been used to monitor ferroptotic response under different conditions ${ }^{59,69}$. However, the specific quantitative markers of ferroptotic death in vivo have not yet been determined. Given that LCN2 plays a wide role in preventing infection and sterile inflammation in various animal models ${ }^{51-53}$, LCN2-mediated ferroptosis resistance may be a common mechanism driving cell protection and reducing morbidity and mortality, a possibility that awaits further investigation in future studies.

In summary, our studies reveal a potential negative role for NUPR1 in ferroptosis. The crosstalk between gene transcription and iron metabolism may have broad implications for modulating ferroptosis in pathologic conditions.

\section{Methods}

Reagents. Erastin (S7242), ferrostatin-1 (S7243), liproxstatin-1 (S7699), RSL3 (S8155), IKE (S8877), DFO (S5742), NAC (S5804), Z-VAD-FMK (S7023), and necrosulfonamide (S8251) were purchased from Selleck Chemicals. L-arginine (A5131) was purchased from Sigma-Aldrich. ZZW-115 (PC-36152) was purchased from ProbeChem. The antibody to ACTB (3700; RRID:AB_2242334; 1:1000) was obtained from Cell Signaling Technology. The antibody to LCN2 (ab63929; RRID: AB_1140965; 1:500) was obtained from Abcam. The antibody to NUPR1 (SAB2109172 [RRID:AB_2868575; 1:500] or sc-23283 [RRID:AB_2157971; 1:100]) was obtained from Sigma-Aldrich or Santa Cruz Biotechnology.

Cell culture. The PANC1 (CRL-1469) and MIAPaCa2 (CRL-1420) cell lines were obtained from the American Type Culture Collection. PHsPDAC cells were a gift from Yangchun Xie and they were generated from patient tumor specimens ${ }^{70,71}$. WT and Nupr1 $1^{-1-}$ mPDAC cells were generated from Nupr $1^{+/+} ; P d x 1-c r e ; L S L-$ Kras $^{G 12 D}$ or Nupr1 $1^{-/-} ; P d x 1-c r e ; L S L-K r a s^{G 12 D}$ mice, respectively, which was a gift from Juan Iovanna (Centre de Recherche en Cancérologie de Marseille, INSERM, France). WT and Nupr $1^{-1-}$ mPDAC were used at $<10$ passages. These cells were cultured in Dulbecco's modified Eagle's medium (Thermo Fisher Scientific, 11995073 ) supplemented with $10 \%$ heat-inactivated fetal bovine serum (Thermo Fisher Scientific, A3840001) and $1 \%$ penicillin and streptomycin (Thermo Fisher Scientific, $15070-063$ ) at $37^{\circ} \mathrm{C}, 95 \%$ humidity, and $5 \% \mathrm{CO}_{2}$. Cell line identity was validated by short tandem repeat profiling, and routine mycoplasma testing was negative for contamination.

Cell viability assay. Cell viability was assayed by a CCK8 kit (Dojindo Laboratories, CK04). In brief, cells were seeded into 96-well plates and incubated with the indicated treatments. Subsequently, $100 \mu \mathrm{l}$ of fresh medium was added to cells containing $10 \mu \mathrm{l}$ of CCK- 8 solutions and incubated for $2 \mathrm{~h}\left(37^{\circ} \mathrm{C}, 5 \% \mathrm{CO}_{2}\right)$. Absorbance at $450 \mathrm{~nm}$ was measured using a microplate reader (Cytation 5 Cell Imaging Multi-Mode Reader). In addition, a Countess II FL Automated Cell Counter (Thermo Fisher Scientific) was used to assay the percentages of dead cells after cell staining with $0.4 \%$ trypan blue solution (Thermo Fisher Scientific, $\mathrm{T} 10282)$

RNAi and gene transfection. Mouse Nupr1-cDNA (EX-Mm07534-M02) or Lcn2 cDNA (EX-Mm03601-M02) or or Atf4-cDNA (EX-Mm20335-M02) were purchased from GeneCopoeia. Human NUPR1-shRNA (sequence: CCGGGGATGAATCTGACCTCTATAGCTCGAGCTATAGAGGTCAGATTCATCCTTTT TG), human LCN2-shRNA (sequence: CCGGGCTGGGCAACATTAAGAGTTAC TCGAGTAACTCTTAATGTTGCCCAGCTTTTTG), human ATF4-shRNA-1 (sequence: CCGGGCCTAGGTCTCTTAGATGATTCTCGAGAATCATCTAAGA GACCTAGGCTTTTT), human ATF4-shRNA-2 (sequence: CCGGGTTGGTCA GTCCCTCCAACAACTCGAGTTGTTGGAGGGACTGACCAACTTTTT), mouse Lcn2-shRNA (sequence: CCGGCCAGGACTCAACTCAGAACTTCTCGA GAAGTTCTGAGTTGAGTCCTGGTTTTTG), mouse Steap3-shRNA-1 (sequence: CCGGACAGCGAGGTGATGATATATGCTCGAGCATATATCATC ACCTCGCTGTTTTTTG), and mouse Steap3-shRNA-2 (sequence: CCGGCGC TCCCGTCCATTGCTAATTCTCGAGAATTAGCAATGGACGGGAGCGTTTT TG) were obtained from Sigma-Aldrich. Transfection with shRNA or cDNA was performed with Lipofectamine 3000 (Invitrogen, L3000-015) according to the manufacturer's instructions.

NanoString nCounter analysis. Total RNA from tumors was extracted using the RNeasy Plus Mini Kit (QIAGEN, 74136) and hybridized with the NanoString nCounter Human PanCancer Pathways Panel Code Set (NanoString) with 770 genes from 13 cancer-associated canonical pathways, including MAPK, STAT, PI3K, RAS, cell cycle, apoptosis, Hedgehog, Wnt, DNA damage control, transcriptional regulation, chromatin modification, and TGF- $\beta^{19}$. Briefly, 100 ng of scaled RNA was hybridized with biotin-labeled capture probes and fluorescently labeled reporter probes for $18 \mathrm{~h}$ at $65^{\circ} \mathrm{C}$. Subsequently, the strip tubes were placed into the nCounter Prep Station for automated sample purification and subsequent reporter capture. Each sample was scanned for 600 FOV on the nCounter Digital Analyzer. NanoString results were produced from RCC files using nSolver Analysis Software 3.0.

qPCR analysis. Total RNA was extracted and purified from cultured cells or tissues using the RNeasy Plus Mini Kit (QIAGEN, 74136) according to the manufacturer's instructions. The RNA was quantified by determining absorbance at $260 \mathrm{~nm}$. One microgram of total RNA from each sample was reverse-transcribed into cDNA 
using the iScript cDNA synthesis kit (Bio-Rad, 170-8891) in a volume of $20 \mu \mathrm{l}$; cDNA from cell samples was amplified. The qPCR was performed using SsoFast EvaGreen Supermix (Bio-Rad, 172-5204) on the C1000 Touch Thermocycler CFX96 Real-Time System (Bio-Rad) according to the manufacturer's protocol. Analysis was performed using Bio-Rad CFX Manager software 3.1 (Bio-Rad). The specific primers were listed in Supplementary Table 1 . The gene expression was calculated via the $2^{-\Delta \Delta \mathrm{Ct}}$ method and normalized to $18 S R N A / 18 s r n a^{72}$. The relative concentrations of mRNA were expressed in arbitrary units based on the untreated group, which was assigned a value of 1 .

Western blot. Cells were lysed in $1 \times$ cell lysis buffer (Cell Signaling Technology, 9803) containing protease inhibitor (ROCHE, 11836153001) on ice for $10 \mathrm{~min}$. After centrifugation at $14,000 \times g$ for $15 \mathrm{~min}$ at $4{ }^{\circ} \mathrm{C}$, the supernatants were collected and quantified using BCA assay (Thermo Fisher Scientific, 23225). The $30 \mu \mathrm{g}$ of each sample was resolved on $4-12 \%$ Criterion XT Bis-Tris gels (Bio-Rad, 3450124) in XT MES running buffer (Bio-Rad, 1610789) and transferred to PVDF membranes (Bio-Rad, 1620233) using the Trans-Blot Turbo Transfer Pack and System (Bio-Rad) ${ }^{73}$. After blocking by TBST containing $5 \%$ skim milk for $1 \mathrm{~h}$, the membrane was incubated overnight at $4{ }^{\circ} \mathrm{C}$ with various primary antibodies (1:200-1:1000). After incubation with peroxidase-conjugated secondary antibodies (goat anti-rabbit IgG secondary antibody [Cell Signaling Technology, 7074, RRID: AB_2099233, 1:1000]; horse anti-mouse IgG secondary antibody [Cell Signaling Technology, 7076, RRID:AB_330924, 1:1000]; rabbit anti-goat IgG secondary antibody [Abcam, ab6741, RRID:AB_955424, 1:1000]) for $1 \mathrm{~h}$ at room temperature, the signals were visualized using enhanced chemiluminescence (Thermo Fisher Scientific, 34095). We collected protein from each cell line in three biologically independent samples and mixed them together for western blot analysis, and repeated twice. The relative intensities of the bands of western blots from three regions were automatically analyzed and normalized to a loading control using the ChemiDoc Touch Imaging System Version 1.2 (Bio-Rad). Source data of images of western blot bands are provided as a Source Data file.

Biochemical assay. Commercially available enzyme-linked immunosorbant assay (ELISA) kits were used to measure the concentrations or activity of HMGB1 (Shino Test Corporation, ST51011), amylase (BioVision, K711), MPO (BioVision, K744), iron (Abcam, ab83366), MDA (Abcam, ab118970), and 8-OHdG (Cell Biolabs, STA-320) in indicated samples according to the manufacturer's instructions. Data were normalized to protein or DNA concentration. In addition, C11-BODIPY probe (Thermo Fisher Scientific, D3861) was used to detect lipid ROS in cells.

Secrete-pair luminescence and ChIP assay. Dual-reporter promoter clones or controls were transfected into two cell lines in duplicates. Indicated WT and Nupr1 $1^{-1-}$ cells were transfected with pEZX-PG04-Lcn2 promoter Gaussia luciferase/secreted alkaline phosphatase (GeneCopoeia, MPRM39850-PG04). After 48 $\mathrm{h}$, these cells were treated with erastin $(10 \mu \mathrm{M})$ or RSL3 $(1 \mu \mathrm{M})$ at indicated times. The $L c n 2$ promoter luciferase activity was measured with a Secrete-Pair Dual Luminescence Assay Kit (GeneCopoeia, SPDA-D010) in accordance with the manufacturer's guidelines. The chromatin immunoprecipitation (ChIP) assay was performed using the Pierce Magnetic ChIP Kit (Thermo Scientific, 26157). This kit contained reagents to lyse cells and extract and solubilize the crosslinked complexes. The complexes were then incubated with anti-NUPR1 antibody (Santa Cruz Biotechnology, sc-23283) and isolated using Pierce Protein A/G Magnetic Beads. After reversing crosslinks and digesting protein, the resulting DNA fragments were purified. One-twentieth of the immunoprecipitated DNA was used in qPCR. The results were shown as a percentage of input.

Animal model. We conducted all animal care and experiments in accordance with the Association for Assessment and Accreditation of Laboratory Animal Care guidelines and with approval from our Institutional Animal Care and Use Committee (Guangzhou Medical University [\#2019075] and UT Southwestern Medical Center [\#102605]). All mice were housed under a 12-h light-dark diurnal cycle with controlled temperature $\left(20-25^{\circ} \mathrm{C}\right)$ and relative humidity $(40-60 \%)$. Food and water were available ad libitum. Experiments were carried out under pathogen-free conditions and the health status of mouse lines was routinely checked by veterinary staff. No wild animals were used in the study. Experiments were carried out with randomly chosen littermates of the same sex and matched by age and body weight. Animals were sacrificed at the indicated time by $\mathrm{CO}_{2}$ asphyxia, and blood samples and tissue were collected.

To generate murine subcutaneous tumors, $5 \times 10^{6} \mathrm{PANC1}$ or MIAPaCa2 cells in $100 \mu \mathrm{l}$ PBS was injected subcutaneously to the right of the dorsal midline in 6-8week-old athymic nude female mice. Once the tumors reached around $80 \mathrm{~mm}^{3}$ at day 7 , mice were randomly allocated into groups and then treated with IKE $(40 \mathrm{mg} / \mathrm{kg}$, i.p., once every other day) in the absence or presence of Z-VAD-FMK $(10 \mathrm{mg} / \mathrm{kg}$, i.p., once every other day) or necrosulfonamide $(10 \mathrm{mg} / \mathrm{kg}$, i.p., once every other day) or liproxstatin-1 (10 mg/kg, i.p., once every other day) or ZZW115 or liproxstatin-1 $(5 \mathrm{mg} / \mathrm{kg}$, i.p., once every other day) starting at day 7 for 2 weeks. Tumors were measured twice weekly and volumes were calculated using the formula length $\times$ width $^{2} \times \pi / 6$.
Pancreatic-specific $L c n 2$-knockout mice were generated by crossing floxed $L c n 2$

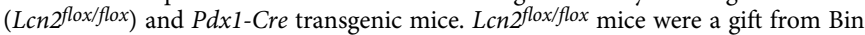
Gao (National Institutes of Health, USA). Pdx1-Cre mice (014647) were purchased from the Jackson Laboratory. All mice were C57BL/6 background. For L-arginineinduced pancreatitis, a sterile solution of L-arginine monohydrochloride (8\%) was prepared in normal saline and the $\mathrm{pH}$ was adjusted to 7.0. Mice received two hourly intraperitoneal (i.p.) injections of L-arginine $(4 \mathrm{~g} / \mathrm{kg})$, while controls were administered saline i.p. ${ }^{74}$. In addition, pretreatment with liproxstatin-1 $(10 \mathrm{mg} / \mathrm{kg})$ or DFO $(100 \mathrm{mg} / \mathrm{kg})$ for $1 \mathrm{~h}$ was used in pancreatitis models.

Bioinformatics analysis. GEPIA (http://gepia.cancer-pku.cn/index.html) ${ }^{75}$, an interactive web server for analyzing the TCGA data, was used to separate the TCGA cohorts into groups with high/low expression of selected genes, which were then used for the prognostic signature validation based on the best cut-off values The best expression cut-off refers the fragments per kilobase of exon model per million reads mapped (FPKM) value that yields maximal difference with regard to survival between the two groups at the lowest log-rank $P$-value.

Statistical analysis. Data are presented as mean \pm SD except where otherwise indicated. GraphPad Prism 8.4.3 was used to collect and analyze data. Unpaired Student's $t$ tests were used to compare the means of two groups. A one-way (for one independent variable) or two-way (for two independent variables) analysis of variance (ANOVA) with Tukey's multiple comparisons test was used for comparison among the different groups on all pairwise combinations. Log-rank test was used to compare differences in mortality rates between groups. A two-tailed $P$ value of $<0.05$ was considered statistically significant. The exact value of $n$ within the figures and replicates is indicated in the figure legends. We did not exclude samples or animals. No statistical methods were used to predetermine sample sizes, but our sample sizes are similar to those generally employed in the field ${ }^{26,76}$.

Reporting summary. Further information on research design is available in the Nature Research Reporting Summary linked to this article.

\section{Data availability}

All the other data supporting the findings of this study are available within the article and its supplementary information files and from the corresponding author upon reasonable request GEPIA (http://gepia.cancer-pku.cn/index.html) ${ }^{75}$ was used to analyze the TCGA data of NURP1 (Ensembl ID: ENSG00000176046 [https://www.ncbi.nlm.nih.gov/gene/26471]) or LCN2 (Ensembl ID: ENSG00000148346 [https://www.ncbi.nlm.nih.gov/gene/3934]) gene. Source data are provided with this paper.

\section{Received: 1 April 2020; Accepted: 24 December 2020;}

Published online: 28 January 2021

\section{References}

1. Galluzzi, L. et al. Molecular mechanisms of cell death: recommendations of the Nomenclature Committee on Cell Death 2018. Cell Death Differ. 25, 486-541 (2018).

2. Tang, D., Kang, R., Berghe, T. V., Vandenabeele, P. \& Kroemer, G. The molecular machinery of regulated cell death. Cell Res. 29, 347-364 (2019).

3. Dixon, S. J. et al. Ferroptosis: an iron-dependent form of nonapoptotic cell death. Cell 149, 1060-1072 (2012).

4. Tang, D. \& Kroemer, G. Ferroptosis. Curr. Biol. 30, R1292-R1297 (2020).

5. Xie, Y. et al. Ferroptosis: process and function. Cell Death Differ. 23, 369-379 (2016).

6. Stockwell, B. R. et al. Ferroptosis: a regulated cell death nexus linking metabolism, redox biology, and disease. Cell 171, 273-285 (2017).

7. Tang, D., Chen, X., Kang, R. \& Kroemer, G. Ferroptosis: molecular mechanisms and health implications. Cell Res. https://doi.org/10.1038/s41422 41020-00441-41421 (2020).

8. Chen, X., Yu, C., Kang, R. \& Tang, D. Iron metabolism in ferroptosis. Front Cell Dev. Biol. 8, 590226 (2020).

9. Wen, Q., Liu, J., Kang, R., Zhou, B. \& Tang, D. The release and activity of HMGB1 in ferroptosis. Biochem. Biophys. Res. Commun. 510, 278-283 (2019).

10. Chen, X., Li, J., Kang, R., Klionsky, D. J. \& Tang, D. Ferroptosis: machinery and regulation. Autophagy, 1-28 https://doi.org/10.1080/15548627.2020.1810918 (2020)

11. Dai, C. et al. Transcription factors in ferroptotic cell death. Cancer Gene Ther 27, 645-656 (2020).

12. Sun, X. et al. Activation of the p62-Keap1-NRF2 pathway protects against ferroptosis in hepatocellular carcinoma cells. Hepatology 63, 173-184 (2016).

13. Anandhan, A., Dodson, M., Schmidlin, C. J., Liu, P. \& Zhang, D. D. Breakdown of an ironclad defense system: the critical role of NRF2 in 
mediating ferroptosis. Cell Chem. Biol. https://doi.org/10.1016/j. chembiol.2020.03.011 (2020).

14. Sun, X. et al. Metallothionein- $1 \mathrm{G}$ facilitates sorafenib resistance through inhibition of ferroptosis. Hepatology 64, 488-500 (2016).

15. Xie, Y. C. et al. The tumor suppressor p53 limits ferroptosis by blocking DPP4 activity. Cell Rep. 20, 1692-1704 (2017).

16. Jiang, L. et al. Ferroptosis as a p53-mediated activity during tumour suppression. Nature 520, 57-62 (2015).

17. Kang, R., Kroemer, G. \& Tang, D. The tumor suppressor protein p53 and the ferroptosis network. Free Radic. Biol. Med. 133, 162-168 (2019).

18. Kuang, F., Liu, J., Tang, D. \& Kang, R. Oxidative damage and antioxidant defense in ferroptosis. Front. Cell Dev. Biol. 8, 586578 (2020).

19. Geiss, G. K. et al. Direct multiplexed measurement of gene expression with color-coded probe pairs. Nat. Biotechnol. 26, 317-325 (2008).

20. Dixon, S. J. et al. Pharmacological inhibition of cystine-glutamate exchange induces endoplasmic reticulum stress and ferroptosis. Elife 3, e02523 (2014).

21. Chen, D. et al. ATF4 promotes angiogenesis and neuronal cell death and confers ferroptosis in a xCT-dependent manner. Oncogene 36, 5593-5608 (2017).

22. Zhu, S. et al. HSPA5 regulates ferroptotic cell death in cancer cells. Cancer Res. 77, 2064-2077 (2017).

23. Song, X. et al. FANCD2 protects against bone marrow injury from ferroptosis. Biochem. Biophys. Res. Commun. 480, 443-449 (2016).

24. Yang, W. S. et al. Peroxidation of polyunsaturated fatty acids by lipoxygenases drives ferroptosis. Proc. Natl Acad. Sci. USA 113, E4966-E4975 (2016).

25. Torti, S. V. \& Torti, F. M. Iron and cancer: more ore to be mined. Nat. Rev. Cancer 13, 342-355 (2013).

26. Zhang, Y. et al. Imidazole ketone erastin induces ferroptosis and slows tumor growth in a mouse lymphoma model. Cell Chem. Biol. 26, 623-633 e629 (2019).

27. Yang, W. S. et al. Regulation of ferroptotic cancer cell death by GPX4. Cell 156, 317-331 (2014).

28. Santofimia-Castano, P. et al. Ligand-based design identifies a potent NUPR1 inhibitor exerting anticancer activity via necroptosis. J. Clin. Investig. 129, 2500-2513 (2019).

29. Ganz, T. \& Nemeth, E. Iron homeostasis in host defence and inflammation. Nat. Rev. Immunol. 15, 500-510 (2015).

30. Liu, Y., Wang, Y., Liu, J., Kang, R. \& Tang, D. The circadian clock protects against ferroptosis-induced sterile inflammation. Biochem. Biophys. Res. Commun. 525, 620-625 (2020)

31. Hamidi, T. et al. Nuclear protein 1 promotes pancreatic cancer development and protects cells from stress by inhibiting apoptosis. J. Clin. Investig. 122, 2092-2103 (2012).

32. Gomez-Chou, S. B. et al. Lipocalin-2 promotes pancreatic ductal adenocarcinoma by regulating inflammation in the tumor microenvironment. Cancer Res. 77, 2647-2660 (2017).

33. Lambert, S. A. et al. The human transcription factors. Cell 172, 650-665 (2018).

34. Mallo, G. V. et al. Cloning and expression of the rat $\mathrm{p} 8 \mathrm{cDNA}$, a new gene activated in pancreas during the acute phase of pancreatitis, pancreatic development, and regeneration, and which promotes cellular growth. J. Biol. Chem. 272, 32360-32369 (1997)

35. Clark, D. W. et al. NUPR1 interacts with $\mathrm{p} 53$, transcriptionally regulates $\mathrm{p} 21$ and rescues breast epithelial cells from doxorubicin-induced genotoxic stress. Curr. Cancer Drug Targets 8, 421-430 (2008).

36. Gironella, M. et al. p8/nupr1 regulates DNA-repair activity after doublestrand gamma irradiation-induced DNA damage. J. Cell. Physiol. 221 , 594-602 (2009).

37. Aguado-Llera, D. et al. Deciphering the binding between Nupr1 and MSL1 and their DNA-repairing activity. PLoS ONE 8, e78101 (2013).

38. Ghandhi, S. A. et al. RAD9 deficiency enhances radiation induced bystander DNA damage and transcriptomal response. Radiat. Oncol. 9, 206 (2014).

39. Vasseur, S. et al. Structural and functional characterization of the mouse $\mathrm{p} 8$ gene: promotion of transcription by the CAAT-enhancer binding protein alpha (C/EBPalpha) and C/EBPbeta trans-acting factors involves a C/EBP cisacting element and other regions of the promoter. Biochem. J. 343, 377-383 (1999).

40. Santofimia-Castano, P. et al. Targeting the stress-induced protein NUPR1 to treat pancreatic adenocarcinoma. Cells 8, https://doi.org/10.3390/cells8111453 (2019).

41. Hamidi, T. et al. Nupr1-aurora kinase A pathway provides protection against metabolic stress-mediated autophagic-associated cell death. Clin. Cancer Res. 18, 5234-5246 (2012).

42. Palam, L. R., Gore, J., Craven, K. E., Wilson, J. L. \& Korc, M. Integrated stress response is critical for gemcitabine resistance in pancreatic ductal adenocarcinoma. Cell Death Dis. 6, e1913 (2015).

43. Narzt, M. S. et al. A novel role for NUPR1 in the keratinocyte stress response to UV oxidized phospholipids. Redox Biol. 20, 467-482 (2019).
44. Santofimia-Castano, P. et al. Inactivation of NUPR1 promotes cell death by coupling ER-stress responses with necrosis. Sci. Rep. 8, 16999 (2018).

45. Huang, J. et al. Progressive endoplasmic reticulum stress contributes to hepatocarcinogenesis in fatty acyl-CoA oxidase 1-deficient mice. Am. J. Pathol. 179, 703-713 (2011)

46. Jin, H. O. et al. Nuclear protein 1 induced by ATF4 in response to various stressors acts as a positive regulator on the transcriptional activation of ATF4. IUBMB Life 61, 1153-1158 (2009).

47. Chowdhury, U. R., Samant, R. S., Fodstad, O. \& Shevde, L. A. Emerging role of nuclear protein 1 (NUPR1) in cancer biology. Cancer Metastasis Rev. 28, 225-232 (2009).

48. Emma, M. R. et al. NUPR1, a new target in liver cancer: implication in controlling cell growth, migration, invasion and sorafenib resistance. Cell Death Dis. 7, e2269 (2016)

49. Li, A. et al. NUPR1 silencing induces autophagy-mediated apoptosis in multiple myeloma cells through the PI3K/AKT/mTOR pathway. DNA Cell Biol. https://doi.org/10.1089/dna.2019.5196 (2020).

50. Tang, B. et al. Shiga toxins induce autophagic cell death in intestinal epithelia cells via the endoplasmic reticulum stress pathway. Autophagy 11, 344-354 (2015).

51. Flo, T. H. et al. Lipocalin 2 mediates an innate immune response to bacterial infection by sequestrating iron. Nature 432, 917-921 (2004).

52. Xiao, X., Yeoh, B. S. \& Vijay-Kumar, M. Lipocalin 2: an emerging player in iron homeostasis and inflammation. Annu. Rev. Nutr. 37, 103-130 (2017).

53. Asimakopoulou, A., Weiskirchen, S. \& Weiskirchen, R. Lipocalin 2 (LCN2) expression in hepatic malfunction and therapy. Front. Physiol. 7, 430 (2016).

54. Gao, M. et al. Role of mitochondria in ferroptosis. Mol. Cell 73, 354-363 e353 (2019).

55. Li, C. et al. Mitochondrial DNA stress triggers autophagy-dependent ferroptotic death. Autophagy 1-13, https://doi.org/10.1080/15548627.2020.1739447 (2020).

56. Urrutia, P. J., Mena, N. P. \& Nunez, M. T. The interplay between iron accumulation, mitochondrial dysfunction, and inflammation during the execution step of neurodegenerative disorders. Front. Pharm. 5, 38 (2014).

57. NaveenKumar, S. K. et al. The role of reactive oxygen species and ferroptosis in heme-mediated activation of human platelets. ACS Chem. Biol. 13, 1996-2002 (2018)

58. Alvarez, S. W. et al. NFS1 undergoes positive selection in lung tumours and protects cells from ferroptosis. Nature 551, 639-643 (2017).

59. Yuan, H., Li, X., Zhang, X., Kang, R. \& Tang, D. Identification of ACSL4 as a biomarker and contributor of ferroptosis. Biochem. Biophys. Res. Commun. 478, 1338-1343 (2016).

60. Doll, S. et al. ACSL4 dictates ferroptosis sensitivity by shaping cellular lipid composition. Nat. Chem. Biol. 13, 91-98 (2017).

61. Kagan, V. E. et al. Oxidized arachidonic and adrenic PEs navigate cells to ferroptosis. Nat. Chem. Biol. 13, 81-90 (2017).

62. Lee, Y. K. et al. Identification of a mitochondrial defect gene signature reveals NUPR1 as a key regulator of liver cancer progression. Hepatology $\mathbf{6 2}$, 1174-1189 (2015)

63. Cano, C. E. et al. Genetic inactivation of Nupr1 acts as a dominant suppressor event in a two-hit model of pancreatic carcinogenesis. Gut 63, 984-995 (2014).

64. Wang, W. et al. CD8(+) T cells regulate tumour ferroptosis during cancer immunotherapy. Nature 569, 270-274 (2019).

65. Viswanathan, V. S. et al. Dependency of a therapy-resistant state of cancer cells on a lipid peroxidase pathway. Nature 547, 453-457 (2017).

66. Xie, Y., Kuang, F., Liu, J., Tang, D. \& Kang, R. DUSP1 blocks autophagydependent ferroptosis in pancreatic cancer. J. Pancreatol. 3, 154-160 (2020).

67. Dai, E. et al. Autophagy-dependent ferroptosis drives tumor-associated macrophage polarization via release and uptake of oncogenic KRAS protein. Autophagy 16, 2069-2083 (2020).

68. Dai, E. et al. Ferroptotic damage promotes pancreatic tumorigenesis through a TMEM173/STING-dependent DNA sensor pathway. Nat. Commun. 11, 6339 (2020).

69. Feng, H. et al. Transferrin receptor is a specific ferroptosis marker. Cell Rep. 30, 3411-3423 e3417 (2020)

70. Song, X. et al. JTC801 induces pH-dependent death specifically in cancer cells and slows growth of tumors in mice. Gastroenterology 154, 1480-1493 (2018).

71. Huang, J. et al. CDK1/2/5 inhibition overcomes IFNG-mediated adaptive immune resistance in pancreatic cancer. Gut https://doi.org/10.1136/gutjnl2019-320441 (2020).

72. Deng, W. et al. The Circadian clock controls immune checkpoint pathway in sepsis. Cell Rep. 24, 366-378 (2018).

73. Tang, D. et al. Endogenous HMGB1 regulates autophagy. J. Cell Biol. 190, 881-892 (2010).

74. Kang, R. et al. Intracellular Hmgb1 inhibits inflammatory nucleosome release and limits acute pancreatitis in mice. Gastroenterology 146, 1097-1107 (2014).

75. Tang, Z. et al. GEPIA: a web server for cancer and normal gene expression profiling and interactive analyses. Nucleic Acids Res. 45, W98-W102 (2017). 
76. Ding, S. P., Li, J. C. \& Jin, C. A mouse model of severe acute pancreatitis induced with caerulein and lipopolysaccharide. World J. Gastroenterol. 9, 584-589 (2003).

\section{Acknowledgements}

We thank Dave Primm (Department of Surgery, University of Texas Southwestern Medical Center) for his critical reading of the manuscript.

\section{Author contributions}

Experiments were conceived and designed by J.L., X.S., G.K., and D.T. Experiments were performed by J.L., X.S., F.K., Q.Z., Y.X., R.K., and D.T. Data were analyzed by J.L., X.S., G.K., and D.T. The paper was written by D.T. and G.K.

\section{Competing interests}

The authors declare no competing interests.

\section{Additional information}

Supplementary information is available for this paper at https://doi.org/10.1038/s41467021-20904-2.

Correspondence and requests for materials should be addressed to G.K. or D.T.
Peer review information Nature Communications thanks John Neoptolemos and the other, anonymous, reviewer(s) for their contribution to the peer review of this work.

Reprints and permission information is available at http://www.nature.com/reprints

Publisher's note Springer Nature remains neutral with regard to jurisdictional claims in published maps and institutional affiliations.

(c) (i) Open Access This article is licensed under a Creative Commons Attribution 4.0 International License, which permits use, sharing, adaptation, distribution and reproduction in any medium or format, as long as you give appropriate credit to the original author(s) and the source, provide a link to the Creative Commons license, and indicate if changes were made. The images or other third party material in this article are included in the article's Creative Commons license, unless indicated otherwise in a credit line to the material. If material is not included in the article's Creative Commons license and your intended use is not permitted by statutory regulation or exceeds the permitted use, you will need to obtain permission directly from the copyright holder. To view a copy of this license, visit http://creativecommons.org/ licenses/by/4.0/.

(c) The Author(s) 2021 